\title{
Packing chromatic number of subdivisions of cubic graphs
}

\author{
József Balogh * $\quad$ Alexandr Kostochka $^{\dagger} \quad \mathrm{Xujun} \mathrm{Liu}^{\ddagger}$
}

October 9, 2018

\begin{abstract}
A packing $k$-coloring of a graph $G$ is a partition of $V(G)$ into sets $V_{1}, \ldots, V_{k}$ such that for each $1 \leq i \leq k$ the distance between any two distinct $x, y \in V_{i}$ is at least $i+1$. The packing chromatic number, $\chi_{p}(G)$, of a graph $G$ is the minimum $k$ such that $G$ has a packing $k$-coloring. For a graph $G$, let $D(G)$ denote the graph obtained from $G$ by subdividing every edge. The questions on the value of the maximum of $\chi_{p}(G)$ and of $\chi_{p}(D(G))$ over the class of subcubic graphs $G$ appear in several papers. Gastineau and Togni asked whether $\chi_{p}(D(G)) \leq 5$ for any subcubic $G$, and later Brešar, Klavžar, Rall and Wash conjectured this, but no upper bound was proved. Recently the authors proved that $\chi_{p}(G)$ is not bounded in the class of subcubic graphs $G$. In contrast, in this paper we show that $\chi_{p}(D(G))$ is bounded in this class, and does not exceed 8 .
\end{abstract}

Mathematics Subject Classification: 05C15, 05C35.

Key words and phrases: packing coloring, cubic graphs, independent sets.

\section{Introduction}

For a positive integer $i$, a set $S$ of vertices in a graph $G$ is $i$-independent if the distance in $G$ between any two distinct vertices of $S$ is at least $i+1$. In particular, a 1-independent set is simply an independent set.

A packing $k$-coloring of a graph $G$ is a partition of $V(G)$ into sets $V_{1}, \ldots, V_{k}$ such that for each $1 \leq i \leq k$, the set $V_{i}$ is $i$-independent. The packing chromatic number, $\chi_{p}(G)$, of a graph $G$, is the minimum $k$ such that $G$ has a packing $k$-coloring. The notion of packing $k$-coloring was introduced in 2008 by Goddard, Hedetniemi, Hedetniemi, Harris and Rall [16] (under the name broadcast coloring) motivated by frequency assignment problems in broadcast networks. The concept has attracted a considerable attention recently: there are around 30 papers on the topic (see e.g. 1, 3, 4, 5, 6, 7, 8, 9, 10, 11, 12, 13, 22, and references in them). In particular, Fiala and Golovach [10] proved that finding the packing chromatic number of a graph is NP-hard even in the

\footnotetext{
${ }^{*}$ Department of Mathematics, University of Illinois at Urbana-Champaign, IL, USA and Moscow Institute of Physics and Technology, 9 Institutskiy per., Dolgoprodny, Moscow Region, 141701, Russian Federation, jobal@illinois.edu. Research of this author is partially supported by NSF Grant DMS-1500121 and by the Langan Scholar Fund (UIUC).

${ }^{\dagger}$ Department of Mathematics, University of Illinois at Urbana-Champaign, IL, USA and Sobolev Institute of Mathematics, Novosibirsk 630090, Russia, kostochk@math.uiuc.edu. Research of this author is supported in part by NSF grant DMS-1600592 and by grants 18-01-00353A and 16-01-00499 of the Russian Foundation for Basic Research.

${ }^{\ddagger}$ Department of Mathematics, University of Illinois at Urbana-Champaign, IL, USA, xliu150@illinois.edu.
} 
class of trees. Sloper [22] showed that there are graphs with maximum degree 4 and arbitrarily large packing chromatic number. In particular, coloring of graph subdivisions were considered. For a graph $G$, let $D(G)$ denote the graph obtained from $G$ by subdividing every edge.

The questions on how large can $\chi_{p}(G)$ and $\chi_{p}(D(G))$ be if $G$ is a subcubic graph (i.e., a graph with maximum degree at most 3) were discussed in several papers (see [6, 7, 13, 21, 22]). In particular, Gastineau and Togni [13] asked whether $\chi_{p}(D(G)) \leq 5$ for every subcubic graph $G$. Brešar, Klavžar, Rall, and Wash [7] later conjectured this and proved the validity of their conjecture for some special classes of subcubic graphs (e.g., the class of generalized Petersen graph). However, no upper bounds for the whole class of (sub)cubic graphs were proved in either case. Recently, the authors [2] showed that $\chi_{p}(G)$ is not bounded in the class of cubic graphs and that 'many' cubic graphs have 'high' packing chromatic number.

In contrast, in this paper we give the first upper bound on $\chi_{p}(D(G))$ for subcubic $G$ : we show that $\chi_{p}(D(G))$ is bounded by 8 in this class. We will prove the following slightly stronger result.

Theorem 1. For every connected subcubic graph $G$, the graph $D(G)$ has a packing 8-coloring such that color 8 is used at most once.

The theorem will be proved in the language of $S$-colorings introduced in [17] and used in [13, 18].

Definition 2. For a non-decreasing sequence $S=\left(s_{1}, s_{2}, \ldots, s_{k}\right)$ of positive integers, an $S$-coloring of a graph $G$ is a partition of $V(G)$ into sets $V_{1}, \ldots, V_{k}$ such that for each $1 \leq i \leq k$ the distance between any two distinct $x, y \in V_{i}$ is at least $s_{i}+1$.

In particular, a $(1, \ldots, 1)$-coloring is an ordinary coloring, and a $(1,2, \ldots, k)$-coloring is a packing $k$-coloring. For subcubic graphs, Gastineau and Togni [13] proved that they are $(1,1,2,2,2)$ colorable and $(1,2,2,2,2,2,2)$-colorable. We will use the following observation of Gastineau and Togni [13].

Proposition 3 (13] Proposition 1). Let $G$ be a graph and $S=\left(s_{1}, \ldots, s_{k}\right)$ be a non-decreasing sequence of integers. If $G$ is $S$-colorable then $D(G)$ is $\left(1,2 s_{1}+1, \ldots, 2 s_{k}+1\right)$-colorable.

In particular, if $G$ is $(1,1,2,2,3,3)$-colorable, then $D(G)$ has a packing 7 -coloring. In view of this, by a feasible coloring of $G$ we call a coloring of $G$ with colors $1_{a}, 1_{b}, 2_{a}, 2_{b}, 3_{a}, 3_{b}$ such that the distance between any two distinct vertices of color $i_{x}$ is at least $i+1$ for all $1 \leq i \leq 3$ and $x \in\{a, b\}$.

Definition 4. A k-degenerate graph is a graph in which every subgraph has a vertex of degree at most $k$.

In the next two sections we discuss feasible coloring of 2-degenerate subcubic graphs. In Section 2, we will show that if a 2-degenerate subcubic graph $G$ has a feasible coloring $f$ and $v, u$ are vertices of $G$ with degree at most 2 , then we can change $f$ to another feasible coloring with some control on the colors of $v$ and $u$. The long proof of one of the lemmas, Lemma 9, is postponed till the last section. Based on the lemmas of Section 2, in Section 3 we prove the following theorem (that gives a better bound than Theorem 1 but for a more restricted class of graphs).

Theorem 5. Every 2-degenerate subcubic graph $G$ has a feasible coloring. In particular, $D(G)$ has a packing 7-coloring.

In Section 4 we use Theorem 5 and the lemmas in Section 2 to derive Theorem 1. In the final section we present a proof of Lemma 9 . 


\section{Lemmas on feasible coloring}

Definition 6. For a positive integer $s$ and a vertex a in a graph $G$, the ball $B_{G}(a, s)$ in $G$ of radius $s$ with center $a$ is $\left\{v \in V(G): d_{G}(v, a) \leq s\right\}$, where $d_{G}(v, a)$ denotes the distance in $G$ between $v$ and $a$. We abbreviate $B_{G}(a, s)$ to $B(a, s)$ when the graph $G$ is clear from the context.

Definition 7. For a positive integer $k$, a $k$-vertex is a vertex of degree exactly $k$.

For $A=\left\{a_{1}, \ldots, a_{n}\right\} \subseteq V(G)$ and a coloring $f$, by $f(A)$ we mean $\left\{f\left(a_{1}\right), \ldots, f\left(a_{n}\right)\right\}$.

Lemma 8. Let $G$ be a subcubic graph and $f$ be a feasible coloring of $G$. Suppose there are 2-vertices $u, v \in V(G)$ with $f(u)=f(v)=2_{a}$. Let $N(u)=\left\{u_{1}, u_{2}\right\}$ and $N(v)=\left\{v_{1}, v_{2}\right\}$. Then $G$ has a feasible coloring $g$ satisfying one of the following:

(a) $g(u)=2_{a}$ and $g(v) \in\left\{1_{a}, 1_{b}\right\}$ or $g(v)=2_{a}$ and $g(u) \in\left\{1_{a}, 1_{b}\right\}$;

(b) $\{g(u), g(v)\}=\left\{2_{a}, 2_{b}\right\}$;

(c) $\left\{g\left(u_{1}\right), g\left(u_{2}\right)\right\}=\left\{g\left(v_{1}\right), g\left(v_{2}\right)\right\}=\left\{1_{a}, 1_{b}\right\}$, and exactly one of $u, v$ has color $2_{a}$.

Proof. If $\left\{f\left(u_{1}\right), f\left(u_{2}\right)\right\} \neq\left\{1_{a}, 1_{b}\right\}$, then we recolor $u$ with a color $\alpha \in\left\{1_{a}, 1_{b}\right\}-\left\{f\left(u_{1}\right), f\left(u_{2}\right)\right\}$, and $(a)$ holds. Thus by the symmetry between $u$ and $v$ we may assume

$$
f\left(u_{1}\right)=f\left(v_{1}\right)=1_{a} \quad \text { and } \quad f\left(u_{2}\right)=f\left(v_{2}\right)=1_{b} .
$$

Since $f(u)=f(v)=2_{a}, N(u) \cap N(v)=\emptyset$. In other words,

$$
\text { all vertices } u_{1}, u_{2}, v_{1} \text { and } v_{2} \text { are distinct. }
$$

Let $G_{1}$ denote the subgraph of $G$ induced by the vertices of colors $1_{a}$ and $1_{b}$. If $u_{1}$ and $u_{2}$ are in distinct components of $G_{1}$, then after switching the colors in the component of $G_{1}$ containing $u_{2}$, we obtain a coloring contradicting (1). Thus we may assume

$$
G \text { has a } 1_{a}, 1_{b} \text {-colored } u_{1}, u_{2} \text {-path } P_{u} \text { and a } 1_{a}, 1_{b} \text {-colored } v_{1}, v_{2} \text {-path } P_{v} .
$$

Case 1: $u_{1} u_{2} \in E(G)$. If $\left|N\left(u_{1}\right)\right|=3$, then let $u_{3} \in N\left(u_{1}\right)-\left\{u, u_{2}\right\}$. Similarly, if $\left|N\left(u_{2}\right)\right|=3$, then let $u_{4} \in N\left(u_{2}\right)-\left\{u, u_{1}\right\}$. If $2_{b} \notin f\left(N\left(u_{1}\right) \cup N\left(u_{2}\right)\right)$, then after recoloring $u$ with $2_{b}$ we get a coloring satisfying (b). Thus we may assume

$$
\left|N\left(u_{1}\right)\right|=3 \text { and } f\left(u_{3}\right)=2_{b} .
$$

Let $N\left(u_{3}\right) \subseteq\left\{u_{1}, u_{5}, u_{6}\right\}$. If $2_{a} \notin f\left(N\left(u_{3}\right)\right)$, then since $f\left(u_{4}\right) \neq 2_{a}$ (because $d\left(u, u_{4}\right)=2$ ) after switching the colors of $u$ and $u_{1}$ we obtain a coloring satisfying $(a)$. So we may assume $f\left(u_{5}\right)=2_{a}$.

Case 1.1: $\left|N\left(u_{2}\right)\right|<3$ or $f\left(u_{4}\right) \neq 2_{b}$. If $1_{b} \notin f\left(N\left(u_{3}\right)\right)$, then we can recolor $u_{3}$ with $1_{b}$. By the case, we can recolor $u$ with $2_{b}$ to obtain a coloring satisfying $(b)$. So we may assume $f\left(u_{6}\right)=1_{b}$ (See Figure 11). Then the coloring $g$ obtained from $f$ by recoloring $u$ and $u_{3}$ with $1_{a}$ and $u_{1}$ with $2_{b}$ satisfies $(a)$.

Case 1.2: $\left|N\left(u_{2}\right)\right|=3$ and $f\left(u_{4}\right)=2_{b}$. If $u_{4}=u_{3}$, then $N\left(u_{3}\right)=\left\{u_{1}, u_{2}, u_{5}\right\}$. Then $u$ has no vertices of color $3_{a}$ at distance at most 3 , so after recoloring $u$ with $3_{a}$, we obtain a coloring $g$ satisfying (c). Thus, $u_{4} \neq u_{3}$.

Case 1.2.1: $1_{b} \notin f\left(N\left(u_{3}\right)\right)$. We recolor $u_{3}$ with $1_{b}$. If $2_{a} \notin f\left(N\left(u_{4}\right)-u_{2}\right)$, then we recolor $u_{2}$ with $2_{a}$ and $u$ with $1_{b}$ to obtain a coloring satisfying $(a)$. If $1_{a} \notin f\left(N\left(u_{4}\right)-u_{2}\right)$, then we recolor $u_{4}$ with $1_{a}, u_{2}$ with $2_{b}$, and $u$ with $1_{b}$ to obtain a coloring satisfying $(a)$. Thus, we may assume 


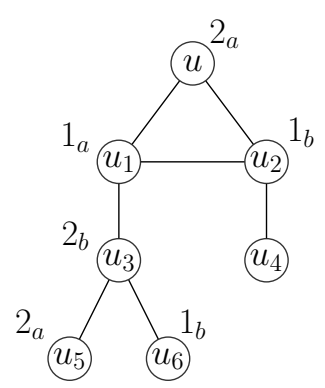

Figure 1: Case 1.1.

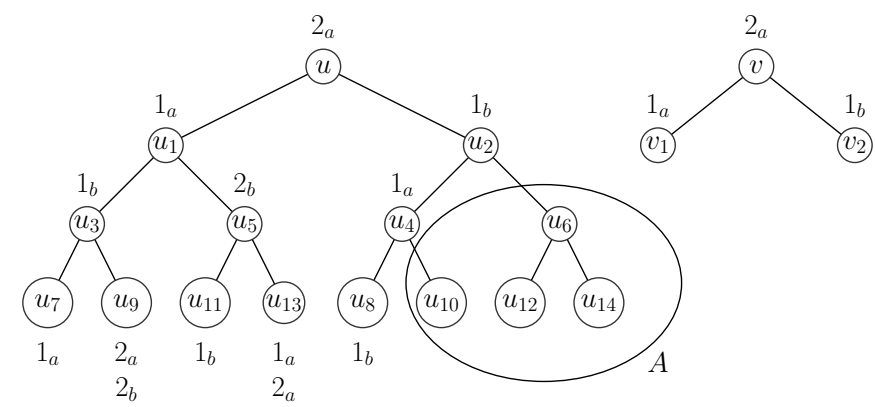

Figure 2: Case 2.1.

$$
f\left(N\left(u_{4}\right)-u_{2}\right)=\left\{1_{a}, 2_{a}\right\} .
$$

Then recoloring $u_{4}$ with $1_{b}, u_{2}$ with $2_{b}$, and $u$ with $1_{b}$, we obtain a coloring satisfying $(a)$.

Case 1.2.2: $1_{b} \in f\left(N\left(u_{3}\right)\right)$. Since $f\left(u_{5}\right)=2_{a}$, this means $u_{6}$ exists and $f\left(u_{6}\right)=1_{b}$. Then we recolor $u_{3}$ and $u_{2}$ with $1_{a}$ and $u_{1}$ with $1_{b}$. If $2_{a} \notin f\left(N\left(u_{4}\right)-u_{2}\right)$, then we recolor $u_{2}$ with $2_{a}$ and $u$ with $1_{a}$ to obtain a coloring satisfying $(a)$. If $1_{b} \notin f\left(N\left(u_{4}\right)-u_{2}\right)$, then we recolor $u_{4}$ with $1_{b}$ and $u$ with $2_{b}$ to obtain a coloring satisfying $(b)$. Thus, we may assume

$$
f\left(N\left(u_{4}\right)-u_{2}\right)=\left\{1_{b}, 2_{a}\right\} .
$$

Then we recolor $u_{4}$ with $1_{a}, u_{2}$ with $2_{b}$, and $u$ with $1_{a}$ to obtain a coloring satisfying $(a)$.

Case 2: $u_{1} u_{2} \notin E(G)$. Then we may assume that $N\left(u_{1}\right) \subseteq\left\{u, u_{3}, u_{5}\right\}, N\left(u_{2}\right) \subseteq\left\{u, u_{4}, u_{6}\right\}$ and by (3),$f\left(u_{3}\right)=1_{b}$ and $f\left(u_{4}\right)=1_{a}$. Furthermore, since by the case, $u_{3} \neq u_{2}$, we may assume that $N\left(u_{3}\right) \subseteq\left\{u_{1}, u_{7}, u_{9}\right\}$ and $f\left(u_{7}\right)=1_{a}$. It is possible that $u_{7}=u_{4}$, but this will not affect the proof below. Similarly, we will assume that $N\left(u_{4}\right) \subseteq\left\{u_{2}, u_{8}, u_{10}\right\}$ and $f\left(u_{8}\right)=1_{b}$. As in Case 1 , $2_{b} \in f\left(N\left(u_{1}\right) \cup N\left(u_{2}\right)\right)$, since otherwise we can recolor $u$ with $2_{b}$ and (b) will hold. In our notation, this means $2_{b} \in\left\{f\left(u_{5}\right), f\left(u_{6}\right)\right\}$. By symmetry, we will assume $f\left(u_{5}\right)=2_{b}$. We also will assume $N\left(u_{5}\right) \subseteq\left\{u_{1}, u_{11}, u_{13}\right\}$ and $N\left(u_{6}\right) \subseteq\left\{u_{2}, u_{12}, u_{14}\right\}$, where some vertices can coincide.

Case 2.1: $\left|N\left(u_{2}\right)\right|<3$ or $f\left(u_{6}\right) \neq 2_{b}$. If $1_{b} \notin f\left(N\left(u_{5}\right)\right)$, then we can recolor $u_{5}$ with $1_{b}$, and then $u$ with $2_{b}$. The resulting coloring satisfies (b). So we may assume $f\left(u_{11}\right)=1_{b}$. If $2_{a} \notin\left\{f\left(u_{9}\right), f\left(u_{13}\right)\right\}$, then by switching the colors of $u$ and $u_{1}$, we obtain a coloring satisfying $(a)$. Thus $2_{a} \in\left\{f\left(u_{9}\right), f\left(u_{13}\right)\right\}$. If $f\left(u_{9}\right)=2_{a}$ and $f\left(u_{13}\right) \neq 1_{a}$ or if $f\left(u_{13}\right)=2_{a}$ and $f\left(u_{9}\right) \neq 2_{b}$, then after switching the colors of $u_{1}$ and $u_{5}$ and recoloring $u$ with $1_{a}$, we again get a coloring satisfying (a). So,

$$
\text { either } f\left(u_{9}\right)=2_{a} \text { and } f\left(u_{13}\right)=1_{a} \text { or } f\left(u_{13}\right)=2_{a} \text { and } f\left(u_{9}\right)=2_{b} \text {. }
$$

If $u_{6}$ does not exist, then by (5), the only vertex in $B(u, 3)-(N(u) \cup\{u\})$ that can be colored with $3_{a}$ or $3_{b}$ is $u_{10}$. Thus after recoloring $u$ with a color in $\left\{3_{a}, 3_{b}\right\}-f\left(u_{10}\right)$ we obtain a coloring satisfying (c). So suppose $u_{6}$ exists. Let $A=\left\{u_{6}, u_{10}, u_{12}, u_{14}\right\}$. If $1_{a} \notin\left\{f\left(u_{12}\right), f\left(u_{14}\right)\right\}$, then we can recolor $u_{6}$ with $1_{a}$ without changing color of any other vertex. Thus we may assume

$$
1_{a} \in f(A) .
$$

If a color $x \in\left\{2_{a}, 2_{b}\right\}$ is not in $f(A)$, then after recoloring $u_{2}$ with $x$ and $u$ with $1_{b}$, we get a coloring satisfying $(a)$. Thus

$$
2_{a}, 2_{b} \in f(A)
$$


By the argument above, in particular, by (5), colors $3_{a}$ and $3_{b}$ are not used on vertices in $B=\left\{u_{1}, u_{2}, u_{3}, u_{4}, u_{5}, u_{7}, u_{8}, u_{9}, u_{11}, u_{13}\right\}$. If at least one of them, say $3_{a}$, is also not used on $A$, then after recoloring $u$ with $3_{a}$, we obtain a coloring satisfying (c). Thus

$$
3_{a}, 3_{b} \in f(A) \text { (See Figure 2). }
$$

Since $|f(A)| \leq 4$, relations (6), (7) and (8) cannot hold at the same time, a contradiction.

Case 2.2: $\left|N\left(u_{2}\right)\right|=3$ and $f\left(u_{6}\right)=2_{b}$. Suppose first that $u_{6}=u_{5}$ and that $N\left(u_{5}\right)=$ $\left\{u_{1}, u_{2}, u_{11}\right\}$. If $f\left(u_{9}\right) \neq 2_{b}$ and $f\left(u_{11}\right) \neq 1_{a}$, then after switching the colors of $u_{1}$ and $u_{5}$ and recoloring $u$ with $1_{a}$, we get a coloring satisfying $(a)$. So, $f\left(u_{9}\right)=2_{b}$ or $f\left(u_{11}\right)=1_{a}$. Similarly, considering switching colors of $u_{2}$ and $u_{5}$, we obtain that $f\left(u_{10}\right)=2_{b}$ or $f\left(u_{11}\right)=1_{b}$. Together, this means

$$
\text { the colors of at least two vertices in }\left\{u_{9}, u_{10}, u_{11}\right\} \text { are in }\left\{1_{a}, 1_{b}, 2_{b}\right\} \text {. }
$$

By (9), some color $y \in\left\{3_{a}, 3_{b}\right\}$ is not used on $B(u, 3)$. Then after recoloring $u$ with $y$, we obtain a coloring satisfying (c).

Now we assume $u_{6} \neq u_{5}$. If $1_{a} \notin\left\{f\left(u_{12}\right), f\left(u_{14}\right)\right\}$, then after recoloring $u_{6}$ with $1_{a}$, we get Case 2.1. Thus below we assume $f\left(u_{12}\right)=1_{a}$. If $2_{a} \notin\left\{f\left(u_{10}\right), f\left(u_{14}\right)\right\}$, then we obtain a coloring satisfying $(a)$ by switching the colors of $u$ and $u_{2}$. Thus, $2_{a} \in\left\{f\left(u_{10}\right), f\left(u_{14}\right)\right\}$. If $f\left(u_{14}\right) \neq 1_{b}$ and $f\left(u_{10}\right) \neq 2_{b}$, then after switching the colors of $u_{2}$ and $u_{6}$ and recoloring $u$ with $1_{b}$, we again get a coloring satisfying $(a)$. So,

$$
\text { either } f\left(u_{10}\right)=2_{a} \text { and } f\left(u_{14}\right)=1_{b} \text { or } f\left(u_{10}\right)=2_{b} \text { and } f\left(u_{14}\right)=2_{a} \text {. }
$$

Let $A=\left\{u_{9}, u_{11}, u_{13}\right\}$. If $2_{a} \notin f(A)$, then we obtain a coloring satisfying $(a)$ by switching the colors of $u$ and $u_{1}$. Thus,

$$
2_{a} \in f(A) .
$$

If $1_{a} \notin f\left(\left\{u_{11}, u_{13}\right\}\right)$ and $f\left(u_{9}\right) \neq 2_{b}$, then after switching the colors of $u_{1}$ and $u_{5}$ and recoloring $u$ with $1_{a}$, we again get a coloring satisfying $(a)$. Therefore,

$$
1_{a} \in f\left(\left\{u_{11}, u_{13}\right\}\right) \text { or } f\left(u_{9}\right)=2_{b} .
$$

By the argument above, in particular, by (10), colors $3_{a}$ and $3_{b}$ are not used on vertices in $B=\left\{u_{1}, u_{2}, u_{3}, u_{4}, u_{5}, u_{7}, u_{8}, u_{10}, u_{12}, u_{14}\right\}$. If at least one of them, say $3_{a}$, is also not used on $A$, then after recoloring $u$ with $3_{a}$, we obtain a coloring satisfying (c). Thus,

$$
3_{a}, 3_{b} \in f(A) .
$$

Since $|f(A)| \leq 3$, relations (11), (12), and (13) cannot hold at the same time, a contradiction.

Our second lemma is:

Lemma 9. Let $G$ be a subcubic graph and $f$ be a feasible coloring of $G$. Suppose there is a 2-vertex $u \in V(G)$ with $N(u)=\left\{u_{1}, u_{2}\right\}$. If $f(u) \in\left\{3_{a}, 3_{b}\right\}$, then we can recolor some vertices of $G$ so that the resulting coloring $g$ is feasible and satisfies the following:

(a) $g(u) \notin\left\{3_{a}, 3_{b}\right\}$, and

(b) at most one vertex is recolored into $3_{a}$ or $3_{b}$, and this vertex (if there is such a vertex) is at distance at most 3 from $u$ and has degree 3 in $G$, and at most one vertex of $f$-color $3_{a}$ or $3_{b}$ apart from $u$ is recolored into some other color, and this vertex (if there is such a vertex) has new color in $\left\{1_{a}, 1_{b}\right\}$.

The proof of this lemma is a long case analysis, so we postpone it to the last section. 


\section{Proof of Theorem 5}

We prove the theorem by induction on the number $n$ of vertices. When $n \leq 6$, the claim holds obviously, since we have 6 colors. When $n>6$, we assume the argument holds for every graph with fewer than $n$ vertices. Let $G$ be any 2-degenerate subcubic graph with $n$ vertices. We may assume $G$ is connected. Since $G$ is 2 -degenerate, it has a vertex, say $w$, with degree at most 2 .

Case 1: $d(w)=1$. Let $N(w)=w^{\prime}$. Since $G-w$ is an $(n-1)$-vertex connected subcubic graph with $d_{G-w}\left(w^{\prime}\right) \leq 2$, by the induction hypothesis, $G-w$ has a $(1,1,2,2,3,3)$-coloring $f$. We color $w$ with a color $x \in\left\{1_{a}, 1_{b}\right\}-f\left(w^{\prime}\right)$ to extend $f$ to $G$.

Case 2: $d(w)=2$. Let $N(w)=\left\{w_{1}, w_{2}\right\}$. Note that $G-w$ has at most two connected components and each connected component is a connected 2-degenerate subcubic graph with less than $n$ vertices. By the induction hypothesis, $G-w$ has a feasible coloring $f$. We may assume that $\left|N_{G-w}\left(w_{1}\right)\right|=\left|N_{G-w}\left(w_{2}\right)\right|=2$. Otherwise we can first apply the induction hypothesis to obtain a $(1,1,2,2,3,3)$-coloring $f$ on $G-w$, then add leaves (vertices of degree one) to $w_{1}$ and $w_{2}$ to obtain a new graph $G^{\prime}$ with $\left|N_{G^{\prime}-w}\left(w_{1}\right)\right|=\left|N_{G^{\prime}-w}\left(w_{2}\right)\right|=2$, then assign proper colors to those leaves we just added to obtain a $(1,1,2,2,3,3)$-coloring $f^{\prime}$ on $G^{\prime}-w$, then prove that $G^{\prime}$ has a $(1,1,2,2,3,3)$-coloring, which can be used to get our desired coloring on $G$. So below we assume $N\left(w_{1}\right)=\left\{w, w_{3}, w_{4}\right\}$ and $N\left(w_{2}\right)=\left\{w, w_{5}, w_{6}\right\}$.

By Lemma 9, $G-w$ has a feasible coloring $f_{1}$ such that $f_{1}\left(w_{1}\right) \notin\left\{3_{a}, 3_{b}\right\}$. Then by Lemma 9 again, $G-w$ also has a feasible coloring $f_{2}$ such that $f_{2}\left(w_{2}\right) \notin\left\{3_{a}, 3_{b}\right\}$ and no vertex of degree 2 in $G-w$ changed its color to $3_{a}$ or $3_{b}$. Thus we also have $f_{2}\left(w_{1}\right) \notin\left\{3_{a}, 3_{b}\right\}$. Therefore, $G-w$ has a feasible coloring $f_{2}$ such that $f_{2}\left(w_{1}\right) \notin\left\{3_{a}, 3_{b}\right\}$ and $f_{2}\left(w_{2}\right) \notin\left\{3_{a}, 3_{b}\right\}$.

Case 2.1: Either $f_{2}\left(w_{1}\right) \neq f_{2}\left(w_{2}\right)$ or $f_{2}\left(w_{1}\right)=f_{2}\left(w_{2}\right) \in\left\{1_{a}, 1_{b}\right\}$. If $\left\{f_{2}\left(w_{1}\right), f_{2}\left(w_{2}\right)\right\} \neq\left\{1_{a}, 1_{b}\right\}$, then we extend $f_{2}$ to $G$ by assigning $f_{2}(w)=\alpha \in\left\{1_{a}, 1_{b}\right\}-\left\{f_{2}\left(w_{1}\right), f_{2}\left(w_{2}\right)\right\}$. By the case, if $f_{2}\left(w_{1}\right)=f_{2}\left(w_{2}\right)$, then $f_{2}\left(w_{1}\right)=f_{2}\left(w_{2}\right) \in\left\{1_{a}, 1_{b}\right\}$. Therefore, the extension of $f_{2}$ to $G$ is feasible since we do not introduce new conflicts between $w_{1}$ and $w_{2}$ by adding $w$. Thus, we may assume

$$
f_{2}\left(w_{1}\right)=1_{a} \quad \text { and } \quad f_{2}\left(w_{2}\right)=1_{b}
$$

If $w_{1}$ and $w_{2}$ are in distinct components of the subgraph $G_{2}$ of $G-w$ induced by the vertices of colors $1_{a}$ and $1_{b}$ in $f_{2}$, then after switching the colors $1_{a}$ and $1_{b}$ with each other in the component of $G_{2}$ containing $w_{2}$, we obtain a coloring contradicting (14). Thus we may assume

$$
G-w \text { has a } 1_{a}, 1_{b} \text {-colored } w_{1}, w_{2} \text {-path } P_{w} .
$$

In particular, we may assume $f_{2}\left(w_{3}\right)=1_{b}$ and $f_{2}\left(w_{5}\right)=1_{a}$ (possibly, $w_{3}=w_{2}$ and then $w_{5}=w_{1}$ ).

If $\left\{2_{a}, 2_{b}\right\} \nsubseteq f_{2}\left(N\left(w_{1}\right) \cup N\left(w_{2}\right)-\{w\}\right)$, then we can extend $f_{2}$ to $G$ by assigning $f_{2}(w)=\beta \in$ $\left\{2_{a}, 2_{b}\right\}-f_{2}\left(N\left(w_{1}\right) \cup N\left(w_{2}\right)-\{w\}\right)$. Thus, we may assume

$$
\begin{gathered}
\left|N\left(w_{1}\right)\right|=\left|N\left(w_{2}\right)\right|=3,\left\{2_{a}, 2_{b}\right\} \subseteq f_{2}\left(N\left(w_{1}\right) \cup N\left(w_{2}\right)-\{w\}\right), \text { and by symmetry } \\
f_{2}\left(w_{4}\right)=2_{a} \text { and } f_{2}\left(w_{6}\right)=2_{b} .
\end{gathered}
$$

If $1_{b} \notin f_{2}\left(N\left(w_{4}\right)-w_{2}\right)$, then we can extend $f_{2}$ to a feasible coloring of $G$ by recoloring $w_{4}$ with $1_{b}$ and letting $f_{2}(w)=2_{a}$. By this and the symmetric statement for $w_{6}$ we can assume that

$w_{4}$ has a neighbor $w_{7}$ with $f_{2}\left(w_{7}\right)=1_{b}$ and $w_{6}$ has a neighbor $w_{8}$ with $f_{2}\left(w_{8}\right)=1_{a}$. 
Case 2.1.1: $w_{1} w_{2} \in E(G)$ (i.e., $w_{3}=w_{2}$ and $\left.w_{5}=w_{1}\right)$. If $1_{a} \notin f_{2}\left(N\left(w_{4}\right)-w_{1}\right)$, then we obtain a feasible coloring on $G$ by switching colors of $w_{1}$ and $w_{4}$, assigning $1_{a}$ to $w$, and using $f_{2}$ on other vertices. Therefore, by (18), we may assume $f_{2}\left(N\left(w_{4}\right)-w_{1}\right)=\left\{1_{a}, 1_{b}\right\}$. Similarly, by (18), we may assume $f_{2}\left(N\left(w_{6}\right)-w_{2}\right)=\left\{1_{a}, 1_{b}\right\}$ (See Figure 33). With (14), (17), and the case, $3_{a} \notin f_{2}(B(w, 3)-\{w\})$ and we can extend $f_{2}$ to $G$ by assigning $f_{2}(w)=3_{a}$.

Case 2.1.2: $w_{1} w_{2} \notin E(G)$. If $N\left(w_{3}\right) \cup N\left(w_{4}\right)$ does not contain a vertex $w_{9}$ of color $2_{b}$, then we can recolor $w_{1}$ with $2_{b}$ and color $w$ with $1_{a}$. So we may assume that $N\left(w_{3}\right) \cup N\left(w_{4}\right)$ contains a vertex $w_{9}$ of color $2_{b}$ and symmetrically $N\left(w_{5}\right) \cup N\left(w_{6}\right)$ contains a vertex $w_{10}$ of color $2_{a}$. Furthermore, if $1_{a} \notin f_{2}\left(N\left(w_{4}\right)-w_{1}\right)$ and $2_{a} \notin f_{2}\left(N\left(w_{3}\right)-w_{1}\right)$, then we can recolor $w_{1}$ with $2_{a}$ and color $w$ and $w_{4}$ with $1_{a}$. With (15) and (18), all vertices in $B\left(w_{1}, 2\right)-w$ have colors in $\left\{1_{a}, 1_{b}, 2_{a}, 2_{b}\right\}$. Symmetrically, we can assume all vertices in $B\left(w_{2}, 2\right)-w$ have colors in $\left\{1_{a}, 1_{b}, 2_{a}, 2_{b}\right\}$ (See Figure 4). Then we can color $w$ with $3_{a}$.

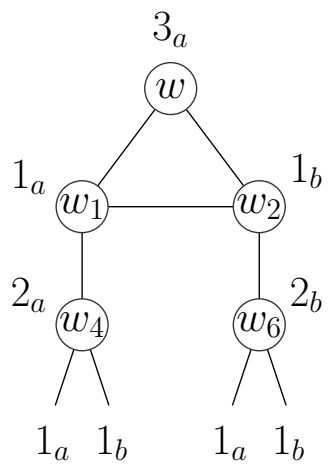

Figure 3: Case 2.1.1.

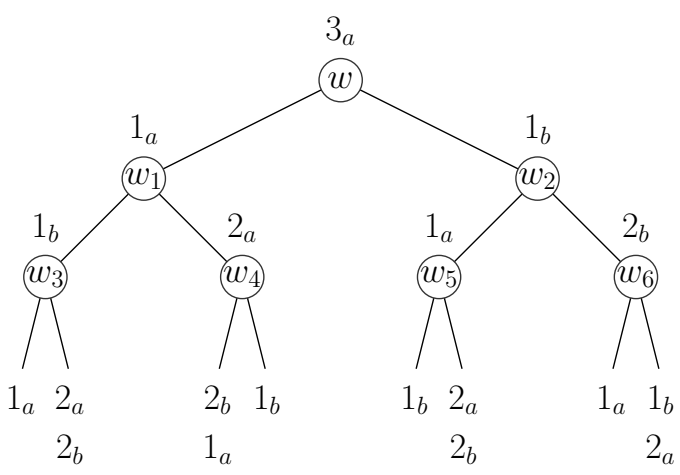

Figure 4: Case 2.1.2.

By the choice of $f_{2}$ and the symmetry of $2_{a}$ and $2_{b}$, the remaining case is:

Case 2.2: $f_{2}\left(w_{1}\right)=f_{2}\left(w_{2}\right)=2_{a}$. In particular, this means $w_{1} w_{2} \notin E(G)$. By Lemma $8, G-w$ has a coloring $g$ satisfying one of the following:

(a) $g\left(w_{1}\right)=2_{a}$ and $g\left(w_{2}\right) \in\left\{1_{a}, 1_{b}\right\}$ or $g\left(w_{2}\right)=2_{a}$ and $g\left(w_{1}\right) \in\left\{1_{a}, 1_{b}\right\}$;

(b) $\left\{g\left(w_{1}\right), g\left(w_{2}\right)\right\}=\left\{2_{a}, 2_{b}\right\}$;

(c) $\left\{g\left(w_{3}\right), g\left(w_{4}\right)\right\}=\left\{g\left(w_{5}\right), g\left(w_{6}\right)\right\}=\left\{1_{a}, 1_{b}\right\}$, and exactly one of $w_{1}, w_{2}$ has color $2_{a}$.

If (a) or (b) occurs, then we again get Case 1. We do not get Case 1 only if (c) occurs and one of $w_{1}, w_{2}$ has $g$-color in $\left\{3_{a}, 3_{b}\right\}$. But then $2_{b}$ is not present in $B(w, 2)$ and we can color $w$ with $2_{b}$.

\section{Cubic graphs}

A good coloring is a $(1,1,2,2,3,3,4)$-coloring with color 4 used at most once. By Proposition 3 , Theorem 1 follows from the following fact.

Theorem 10. Every connected cubic graph has a good coloring.

Proof. Let $G$ be a connected cubic graph with $n \geq 2$ vertices. Since $G$ is connected, it has a non-cut vertex $w$ (simply take a leaf vertex of a spanning tree of $G$ ). Let $N(w)=\left\{w_{1}, w_{2}, w_{3}\right\}$.

Case 1: $0 \leq\left|E\left(G\left[\left\{w_{1}, w_{2}, w_{3}\right\}\right]\right)\right| \leq 1$. If $\left|E\left(G\left[\left\{w_{1}, w_{2}, w_{3}\right\}\right]\right)\right|=0$, then let $G^{\prime}=G-w+w_{2} w_{3}$. If $\left|E\left(G\left[\left\{w_{1}, w_{2}, w_{3}\right\}\right]\right)\right|=1$, then by symmetry we may assume $w_{2} w_{3} \in E(G)$. Let $G^{\prime}=G-w$. 
Note that $G^{\prime}$ is a connected subcubic graph with vertex $w_{1}$ of degree at most two. By Theorem 5 , $G^{\prime}$ has a feasible coloring. Hence by Lemma 9, $G^{\prime}$ has a feasible coloring $f$ with

$$
f\left(w_{1}\right) \notin\left\{3_{a}, 3_{b}\right\} .
$$

Let $N_{G^{\prime}}\left(w_{1}\right)=\left\{w_{4}, w_{5}\right\}, N_{G^{\prime}}\left(w_{2}\right)=\left\{w_{3}, w_{6}, w_{7}\right\}$, and $N_{G^{\prime}}\left(w_{3}\right)=\left\{w_{2}, w_{8}, w_{9}\right\}$. It is possible that $\left|\left\{w_{4}, w_{5}, w_{6}, w_{7}, w_{8}, w_{9}\right\}\right|<6$, but this will not affect the proof below.

For $j \in\{1,2,3\}$ and $x, y \in V(G)-w$, a $(j, x, y)$-conflict in $(G, f)$ is the situation that $f(x)=$ $f(y) \in\left\{j_{a}, j_{b}\right\}$ and $d_{G}(x, y) \leq j$. If $(G, f)$ has no $(j, x, y)$-conflicts for any $j \in\{1,2,3\}$ and $x, y \in V(G)-w$, then we can extend $f$ to a good coloring of $G$ by letting $f(w)=4$.

Suppose now that $(G, f)$ has a $(j, x, y)$-conflict for some $j \in\{1,2,3\}$ and $x, y \in V(G)-w$ (there could be more than one conflict). Then

$$
d_{G}(x, y) \leq j<d_{G^{\prime}}(x, y) \text {. This means }\{x, y\} \cap\left\{w_{1}, w_{2}, w_{3}\right\} \neq \emptyset \text { and } j \geq 2 .
$$

Since $w_{2} w_{3} \in E\left(G^{\prime}\right)$, (20) yields that in each $(j, x, y)$-conflict, one of $x$ and $y$ is in $\left\{w_{1}, w_{4}, w_{5}\right\}$ and the other is in $\left\{w_{2}, w_{3}, w_{6}, w_{7}, w_{8}, w_{9}\right\}$. By (19), we have the following two cases.

Case 1.1: $f\left(w_{1}\right) \in\left\{1_{a}, 1_{b}\right\}$, say $f\left(w_{1}\right)=1_{a}$. Then each conflict is a $(3, x, y)$-conflict.

Case 1.1.1: There is only one conflict. We may assume it is a $\left(3, w_{4}, w_{2}\right)$-conflict, where $f\left(w_{4}\right)=f\left(w_{2}\right)=3_{a}$. If $f\left(N_{G}\left(w_{2}\right)-w\right) \neq\left\{1_{a}, 1_{b}\right\}$, then we can recolor $w_{2}$ with one of $1_{a}$ and $1_{b}$ and eliminate the conflict. If $f\left(w_{3}\right) \neq 1_{b}$, then we can recolor $w_{4}$ with 4 and color $w$ with $1_{b}$. So we may assume

$$
f\left(N_{G}\left(w_{2}\right)-w\right)=\left\{1_{a}, 1_{b}\right\} \quad \text { and } \quad f\left(w_{3}\right)=1_{b} .
$$

Furthermore, if $f\left(w_{5}\right) \neq 1_{b}$ or $1_{a} \notin f\left(N_{G}\left(w_{3}\right)-w\right)$, then we can recolor $w_{1}$ and $w_{3}$ with the same color $\alpha \in\left\{1_{a}, 1_{b}\right\}$, recolor $w_{4}$ with 4 and color $w$ with $\beta \in\left\{1_{a}, 1_{b}\right\}-\alpha$. Otherwise, some $\gamma \in\left\{2_{a}, 2_{b}\right\}$ is not present on $N\left(w_{3}\right) \cup\left\{w_{5}\right\}$, and by (21) we can recolor $w_{4}$ with 4 and color $w$ with $\gamma$ (See Figure 51).

Case 1.1.2: There are two conflicts. By the case and symmetry, we may assume $f\left(w_{4}\right)=$ $f\left(w_{2}\right)=3_{a}$ and $f\left(w_{5}\right)=f\left(w_{3}\right)=3_{b}$. Applying Lemma 9 to vertex $w_{2}$ and coloring $f$ of $G-w$, we obtain a feasible coloring $g$ of $G-w$ such that $g\left(w_{2}\right)=\gamma \notin\left\{3_{a}, 3_{b}\right\}$ and at most one of $w_{3}, w_{4}, w_{5}$ changed its color.

Case 1.1.2.1: Neither $w_{4}$ nor $w_{5}$ changed its color. Then we color $w_{3}$ with color $4, w$ with a color $\beta \in\left\{1_{a}, 1_{b}\right\}-\gamma, w_{1}$ with a color $\alpha \in\left\{1_{a}, 1_{b}\right\}-\beta$, and use $g$ on other vertices.

Case 1.1.2.2: One vertex of $\left\{w_{4}, w_{5}\right\}$ changed its color. We prove the case when $w_{4}$ changed its color, say $g\left(w_{4}\right)=\beta \in\left\{1_{a}, 1_{b}\right\}$, the case $w_{5}$ changed its color is similar. We may assume that

$$
g\left(w_{2}\right)=\gamma \in\left\{1_{a}, 1_{b}\right\} \text { and } \gamma=\beta,
$$

since otherwise we color $w_{1}$ with a color $\alpha \in\left\{1_{a}, 1_{b}\right\}-\beta$, w with a color $\mu \in\left\{1_{a}, 1_{b}\right\}-\alpha$, $w_{3}$ with color 4 , and use $g$ on other vertices. We may also assume that some vertex, say $w_{6} \in$ $N\left(w_{2}\right)-w$, have color $\delta \in\left\{1_{a}, 1_{b}\right\}-\gamma$, since otherwise we recolor $w_{2}$ with $\delta$ and it contradicts (22). We may also assume that $g\left(\left\{w_{8}, w_{9}\right\}\right)=\left\{1_{a}, 1_{b}\right\}$, since otherwise we color $w_{3}$ with a color $\mu \in\left\{1_{a}, 1_{b}\right\}-g\left(\left\{w_{8}, w_{9}\right\}\right), w$ with color 4 , and use $f$ on other vertices (See Figure 6 ). Note that $\left|g(N(w) \cup N(N(w))) \cap\left\{2_{a}, 2_{b}\right\}\right| \leq 1$. Then we color $w_{1}$ with a color $\alpha \in\left\{1_{a}, 1_{b}\right\}-\beta, w_{3}$ with color $4, w$ with a color $\lambda \in\left\{2_{a}, 2_{b}\right\}-g(N(w) \cup N(N(w)))$, and use $g$ on other vertices to obtain a good coloring. 


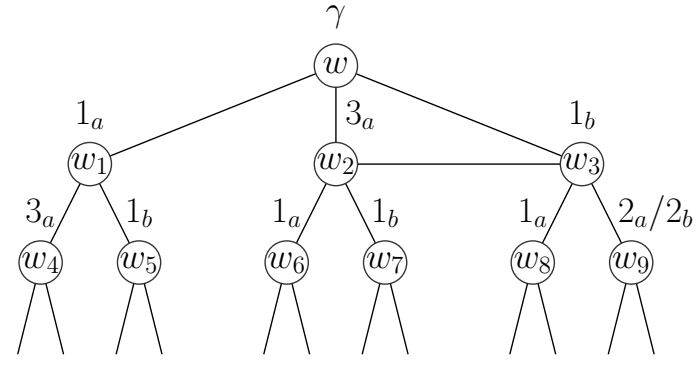

Figure 5: Case 1.1.1.

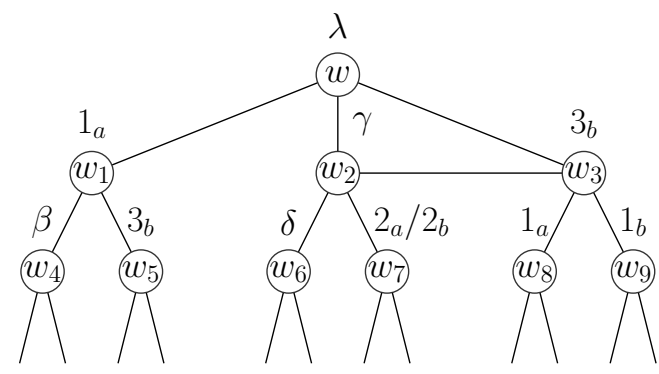

Figure 6: Case 1.1.2.2.

Case 1.2: $f\left(w_{1}\right) \in\left\{2_{a}, 2_{b}\right\}$, say $f\left(w_{1}\right)=2_{a}$. Since we cannot switch to Case 1.1, we need $\left\{f\left(w_{4}\right), f\left(w_{5}\right)\right\}=\left\{1_{a}, 1_{b}\right\}$. So the only possible conflict is a $\left(2, w_{1}, y\right)$-conflict, where $y \in\left\{w_{2}, w_{3}\right\}$. We may assume $f\left(w_{2}\right)=2_{a}$. Then we recolor $w_{1}$ with 4 and color $w$ with $\alpha \in\left\{1_{a}, 1_{b}\right\}-f\left(w_{3}\right)$.

Case 2: $\left|E\left(G\left[\left\{w_{1}, w_{2}, w_{3}\right\}\right]\right)\right|=2$, say $w_{1} w_{2} \in E(G)$ and $w_{2} w_{3} \in E(G)$. We obtain a good coloring $g$ of $G$ by using $f$ on $G-w$ and assigning color 4 to $w$. Note that adding $w$ back will not create conflicts because the distance between any two vertices in $G-w$ remains the same.

Case 3: $G\left[\left\{w_{1}, w_{2}, w_{3}\right\}\right]=K_{3}$. Then $G=K_{4}$, and $K_{4}$ has a good coloring.

\section{Proof of Lemma 9}

Recall the claim of the lemma:

Lemma 9. Let $G$ be a subcubic graph and $f$ be a feasible coloring of $G$. Suppose there is a 2-vertex $u \in V(G)$ with $N(u)=\left\{u_{1}, u_{2}\right\}$. If $f(u) \in\left\{3_{a}, 3_{b}\right\}$, then we can recolor some vertices of $G$ so that the resulting coloring $g$ is feasible and satisfies the following:

(a) $g(u) \notin\left\{3_{a}, 3_{b}\right\}$, and

(b) at most one vertex is recolored into $3_{a}$ or $3_{b}$, and this vertex (if there is such a vertex) is at distance at most 3 from $u$ and has degree 3 in $G$, and at most one vertex of $f$-color $3_{a}$ or $3_{b}$ apart from $u$ is recolored into some other color, and this vertex (if there is such a vertex) has new color in $\left\{1_{a}, 1_{b}\right\}$.

Proof. Without loss of generality, we assume that $f(u)=3_{a}$. If $\left\{f\left(u_{1}\right), f\left(u_{2}\right)\right\} \neq\left\{1_{a}, 1_{b}\right\}$, then we recolor $u$ with a color $x \in\left\{1_{a}, 1_{b}\right\}-\left\{f\left(u_{1}\right), f\left(u_{2}\right)\right\}$ to obtain a coloring satisfying $(a)$ and $(b)$. Thus we may assume

$$
f\left(u_{1}\right)=1_{a} \quad \text { and } \quad f\left(u_{2}\right)=1_{b} .
$$

Let $G_{1}$ denote the subgraph of $G$ induced by the vertices of colors $1_{a}$ and $1_{b}$. If $u_{1}$ and $u_{2}$ are in distinct components of $G_{1}$, then after switching the colors in the component of $G_{1}$ containing $u_{2}$, we obtain a coloring contradicting (23). Thus we may assume

$$
G \text { has a } 1_{a}, 1_{b} \text {-colored } u_{1}, u_{2} \text {-path } P_{u} \text {. }
$$

Case 1: $u_{1} u_{2} \in E(G)$. If $\left|N\left(u_{1}\right)\right|=3$, then let $u_{3} \in N\left(u_{1}\right)-\left\{u, u_{2}\right\}$. Similarly, if $\left|N\left(u_{2}\right)\right|=3$, then let $u_{4} \in N\left(u_{2}\right)-\left\{u, u_{1}\right\}$. If $\left\{2_{a}, 2_{b}\right\} \nsubseteq f\left(N\left(u_{1}\right) \cup N\left(u_{2}\right)\right)$, then after recoloring $u$ with a color 
$x \in\left\{2_{a}, 2_{b}\right\}-f\left(N\left(u_{1}\right) \cup N\left(u_{2}\right)\right)$ we obtain a coloring satisfying $(a)$ and $(b)$. By symmetry, we may assume

$$
\left|N\left(u_{1}\right)\right|=\left|N\left(u_{2}\right)\right|=3, \quad f\left(u_{3}\right)=2_{a} \quad \text { and } \quad f\left(u_{4}\right)=2_{b} .
$$

If $1_{b} \notin f\left(N\left(u_{3}\right)\right)$, then we can recolor $u_{3}$ with $1_{b}$ and $u$ with $2_{a}$ to obtain a coloring satisfying $(a)$ and $(b)$. So we may assume $1_{b} \in f\left(N\left(u_{3}\right)\right)$. Similarly, we may assume $1_{a} \in f\left(N\left(u_{4}\right)\right)$. If $\left|N\left(u_{3}\right)\right|=2$ or $1_{a} \notin f\left(N\left(u_{3}\right)-\left\{u_{1}\right\}\right)$, then we can recolor $u_{3}$ with $1_{a}, u_{1}$ with $2_{a}$, and $u$ with $1_{a}$ to obtain a coloring satisfying $(a)$ and $(b)$. So we may assume

$$
\left|N\left(u_{3}\right)\right|=3 \text { and let } u_{5}, u_{6} \in N\left(u_{3}\right)-\left\{u_{1}\right\} \text { with } f\left(u_{5}\right)=1_{a}, f\left(u_{6}\right)=1_{b} .
$$

Similarly, we may assume

$$
\left|N\left(u_{4}\right)\right|=3 \text { and let } u_{7}, u_{8} \in N\left(u_{4}\right)-\left\{u_{2}\right\} \text { with } f\left(u_{7}\right)=1_{a}, f\left(u_{8}\right)=1_{b} .
$$

Case 1.1: $u_{5}=u_{7}$ and $u_{6}=u_{8}$. If $1_{b} \notin f\left(N\left(u_{5}\right)\right)$, then we can recolor $u_{5}$ with $1_{b}, u_{3}$ with $1_{a}, u_{1}$ with $2_{a}$, and $u$ with $1_{a}$ to obtain a coloring satisfying $(a)$ and $(b)$. So we may assume $1_{b} \in f\left(N\left(u_{5}\right)\right)$. Similarly, we may assume $1_{a} \in f\left(N\left(u_{6}\right)\right)$. Then we can recolor $u_{1}$ with $3_{a}$ and $u$ with $1_{a}$ to obtain a coloring satisfying $(a)$ and $(b)$.

Case 1.2: $u_{5}=u_{7}$ or $u_{6}=u_{8}$, but not both. By symmetry, we may assume $u_{6}=u_{8}$ and $u_{5} \neq u_{7}$. It is possible that $u_{5} u_{6} \in E(G)$ or $u_{6} u_{7} \in E(G)$, but this will not affect the proof below.

Similarly to Case 1.1, we may assume

$$
1_{b} \in f\left(N\left(u_{5}\right)\right), \quad 1_{a} \in f\left(N\left(u_{6}\right)\right) \quad \text { and } \quad 1_{b} \in f\left(N\left(u_{7}\right)\right) .
$$

Since $3_{a} \notin f\left(N\left(u_{6}\right)\right)$, we can also assume $3_{a} \in f\left(N\left(u_{5}\right)\right)$, because otherwise we recolor $u_{1}$ with $3_{a}$ and $u$ with $1_{a}$ to obtain a coloring satisfying $(a)$ and $(b)$. With (25) and (28), we have $f\left(N\left(u_{5}\right)\right)=$ $\left\{1_{b}, 2_{a}, 3_{a}\right\}$. However, we can recolor $u_{1}$ with $3_{b}$ and $u$ with $1_{a}$ to obtain a coloring satisfying $(a)$ and $(b)$.

Case 1.3: $u_{5} \neq u_{7}$ and $u_{6} \neq u_{8}$. Then $N\left(u_{3}\right) \cap N\left(u_{4}\right)=\emptyset$ and $d\left(u_{3}, u_{4}\right) \geq 3$. Similarly to Case $1.2,\left\{1_{a}, 1_{b}, 3_{a}, 3_{b}\right\} \subseteq f\left(N\left(u_{5}\right) \cup N\left(u_{6}\right)-\left\{u_{3}\right\}\right)$ (See Figure 7). Therefore, we can recolor $u_{3}$ with $2_{b}$ and $u$ with $2_{a}$ to obtain a coloring satisfying $(a)$ and $(b)$.

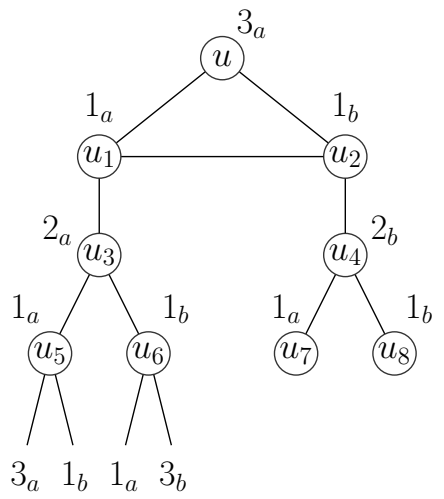

Figure 7: Case 1.3.

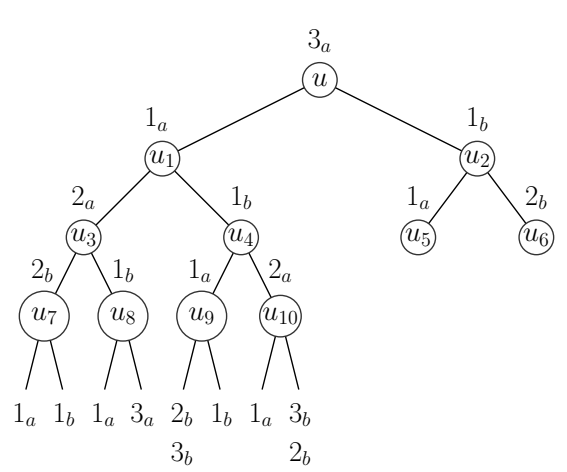

Figure 8: Case 2.1.

Case 2: $u_{1} u_{2} \notin E(G)$. If $\left\{2_{a}, 2_{b}\right\} \nsubseteq f\left(N\left(u_{1}\right) \cup N\left(u_{2}\right)\right)$, then after recoloring $u$ with a color $x \in\left\{2_{a}, 2_{b}\right\}-f\left(N\left(u_{1}\right) \cup N\left(u_{2}\right)\right)$ we obtain a coloring satisfying (a) and (b). With (24), we may 
assume that

$$
\begin{gathered}
N\left(u_{1}\right)=\left\{u, u_{3}, u_{4}\right\}, \quad f\left(u_{3}\right)=2_{a}, \quad f\left(u_{4}\right)=1_{b}, \\
N\left(u_{2}\right)=\left\{u, u_{5}, u_{6}\right\}, \quad f\left(u_{5}\right)=1_{a} \quad \text { and } \quad f\left(u_{6}\right)=2_{b} .
\end{gathered}
$$

If $u_{3} u_{4} \in E(G)$, then $1_{a} \in f\left(N\left(u_{4}\right)-\left\{u_{1}, u_{3}\right\}\right)$ because of (24). We also have $2_{b} \in f\left(N\left(u_{3}\right)-\right.$ $\left.\left\{u_{1}, u_{4}\right\}\right)$ because otherwise we can recolor $u_{1}$ with $2_{b}$ and $u$ with $1_{a}$ to obtain a coloring satisfying $(a)$ and $(b)$. Thus, we may assume $\left|N\left(u_{3}\right)\right|=\left|N\left(u_{4}\right)\right|=3$ and let $u_{7} \in N\left(u_{3}\right)-\left\{u_{1}, u_{4}\right\}, u_{8} \in$ $N\left(u_{4}\right)-\left\{u_{1}, u_{3}\right\}, f\left(u_{7}\right)=2_{b}$, and $f\left(u_{8}\right)=1_{a}$. Then, we can recolor $u_{1}$ with $2_{a}, u_{3}$ with $1_{a}$, and $u$ with $1_{a}$ to obtain a coloring satisfying $(a)$ and $(b)$. Because of symmetry, we may assume

$$
u_{3} u_{4} \notin E(G) \quad \text { and } \quad u_{5} u_{6} \notin E(G) .
$$

If $1_{b} \notin f\left(N\left(u_{3}\right)\right)$, then we recolor $u_{3}$ with $1_{b}$ and $u$ with $2_{a}$ to obtain a coloring satisfying $(a)$ and $(b)$. With (24), we may assume that

$$
1_{b} \in f\left(N\left(u_{3}\right)\right) \quad \text { and } \quad 1_{a} \in f\left(N\left(u_{4}\right)\right) .
$$

If $2_{b} \notin f\left(B\left(u_{1}, 2\right)\right)$, then we can recolor $u_{1}$ with $2_{b}$ and $u$ with $1_{a}$ to obtain a coloring satisfying $(a)$ and $(b)$. Thus, we may assume

$$
2_{b} \in f\left(N\left(u_{3}\right)\right) \cup f\left(N\left(u_{4}\right)\right) .
$$

If $1_{a} \notin f\left(N\left(u_{3}\right)-\left\{u_{1}\right\}\right)$ and $2_{a} \notin f\left(N\left(u_{4}\right)\right)$, then we can recolor $u_{3}$ with $1_{a}, u_{1}$ with $2_{a}$, and $u$ with $1_{a}$ to obtain a coloring satisfying $(a)$ and $(b)$. Thus, we may assume

$$
\left|N\left(u_{3}\right)\right|=\left|N\left(u_{4}\right)\right|=3
$$

and

$$
1_{a} \in f\left(N\left(u_{3}\right)-\left\{u_{1}\right\}\right) \text { or } 2_{a} \in f\left(N\left(u_{4}\right)\right) .
$$

Let $\left\{u_{7}, u_{8}\right\} \in N\left(u_{3}\right),\left\{u_{9}, u_{10}\right\} \in N\left(u_{4}\right)$. By (32), we may assume

$$
f\left(u_{8}\right)=1_{b} \quad \text { and } \quad f\left(u_{9}\right)=1_{a} .
$$

By (33) and (35), we have

$$
\text { either } f\left(u_{7}\right)=2_{b} \text { and } f\left(u_{10}\right)=2_{a} \text { or } f\left(u_{7}\right)=1_{a} \text { and } f\left(u_{10}\right)=2_{b} .
$$

If $3_{a} \notin f\left(B\left(u_{1}, 3\right)-\{u\}\right)$, then we can recolor $u_{1}$ with $3_{a}$ and $u$ with $1_{a}$ to obtain a coloring satisfying $(a)$ and $(b)$. Thus, we may assume

$$
3_{a} \in f\left(B\left(u_{1}, 3\right)-\{u\}\right) .
$$

Similarly, we may assume

$$
3_{b} \in f\left(B\left(u_{1}, 3\right)-\{u\}\right) .
$$

Case 2.1: $f\left(u_{7}\right)=2_{b}$ and $f\left(u_{10}\right)=2_{a}$. By (31) and $\left|N\left(u_{2}\right)\right|=3$, we have

$$
\left\{u_{8}, u_{10}\right\} \cap\left(\left\{u_{i}: i \in[6]\right\} \cup\{u\}\right)=\emptyset .
$$

It is possible that $u_{9}=u_{5}$ or $u_{7}=u_{6}$, but this will not affect the proof below. 
If $2_{b} \notin f\left(B\left(u_{4}, 2\right)\right)$, then we can recolor $u_{4}$ with $2_{b}, u_{1}$ with $1_{b}$, and $u$ with $1_{a}$ to obtain a coloring satisfying $(a)$ and $(b)$. Thus, we may assume

$$
2_{b} \in f\left(B\left(u_{4}, 2\right)\right)
$$

If $1_{a} \notin f\left(N\left(u_{10}\right)\right)$, then we can recolor $u_{10}$ with $1_{a}$ and it contradicts (35). Thus, we may assume

$$
1_{a} \in f\left(N\left(u_{10}\right)\right) \text {. }
$$

We may also assume

$$
f\left(N\left(u_{7}\right)-\left\{u_{3}\right\}\right)=\left\{1_{a}, 1_{b}\right\},
$$

because otherwise we can recolor $u_{7}$ with a color $x \in\left\{1_{a}, 1_{b}\right\}-f\left(N\left(u_{7}\right)-\left\{u_{1}\right\}\right)$ and it contradicts (37). By (38) and (39), we know that

$$
\left\{3_{a}, 3_{b}\right\} \subseteq f\left(N\left(u_{7}\right) \cup N\left(u_{8}\right) \cup N\left(u_{9}\right) \cup N\left(u_{10}\right)\right) .
$$

If $\left\{3_{a}, 3_{b}\right\} \subseteq f\left(N\left(u_{7}\right) \cup N\left(u_{8}\right)\right)$, then by (42) we have $f\left(N\left(u_{8}\right)\right)=\left\{2_{a}, 3_{a}, 3_{b}\right\}$. Then, we can recolor $u_{8}$ with $1_{a}, u_{3}$ with $1_{b}$, and $u$ with $2_{a}$ to obtain a coloring satisfying $(a)$ and $(b)$. By symmetry, we may assume

$$
3_{b} \notin f\left(N\left(u_{7}\right) \cup N\left(u_{8}\right)\right) .
$$

By (43) and (44), we know that $3_{b} \in f\left(N\left(u_{9}\right) \cup N\left(u_{10}\right)\right)$. By (24), $1_{b} \in f\left(N\left(u_{9}\right)-\left\{u_{4}\right\}\right)$. With (40), (41), and $2_{b} \notin f\left(\left\{u, u_{1}, u_{3}, u_{9}, u_{10}\right\}\right)$ we know that

$$
f\left(N\left(u_{9}\right) \cup N\left(u_{10}\right)-\left\{u_{4}\right\}\right)=\left\{1_{a}, 1_{b}, 2_{b}, 3_{b}\right\}, \text { hence } 1_{b} \notin f\left(N\left(u_{10}\right)-\left\{u_{4}\right\}\right) \text { (See Figure } 8 \text { ). }
$$

Therefore, we can recolor $u_{10}$ with $1_{b}, u_{4}$ with $2_{a}, u_{3}$ with $1_{a}, u_{1}$ with $1_{b}$, and $u$ with $1_{a}$ to obtain a coloring satisfying $(a)$ and $(b)$.

Case 2.2: $f\left(u_{7}\right)=1_{a}$ and $f\left(u_{10}\right)=2_{b}$. If $1_{a} \notin f\left(N\left(u_{6}\right)\right)$, then we can recolor $u_{6}$ with $1_{a}$ and $u$ with $2_{b}$ to obtain a coloring satisfying $(a)$ and $(b)$. Thus, we may assume

$$
1_{a} \in f\left(N\left(u_{6}\right)-\left\{u_{2}\right\}\right)
$$

Since some $u_{i}$ and $u_{j}$ may coincide, several cases are considered below.

Case 2.2.1: $u_{3} u_{5} \in E(G)$, i.e., $u_{7}=u_{5}$. It is possible that $u_{4} u_{6} \in E(G)$, or $u_{4} u_{5} \in E(G)$, or $\left\{u_{4} u_{5}, u_{4} u_{6}\right\} \subseteq E(G)$, but this will not affect the proof below. By (24),

$$
1_{b} \in f\left(N\left(u_{9}\right)-\left\{u_{4}\right\}\right)
$$

and

$$
1_{b} \in f\left(N\left(u_{5}\right)-\left\{u_{2}\right\}\right)
$$

If $1_{a} \notin f\left(N\left(u_{10}\right)-\left\{u_{4}\right\}\right)$, then we can recolor $u_{10}$ with $1_{a}$ and it contradicts (37). Thus, we may assume

$$
1_{a} \in f\left(N\left(u_{10}\right)-\left\{u_{4}\right\}\right) .
$$

If $1_{a} \notin f\left(N\left(u_{8}\right)\right)$, then we can recolor $u_{8}$ with $1_{a}, u_{3}$ with $1_{b}$, and $u$ with $2_{a}$ to obtain a coloring satisfying $(a)$ and $(b)$. If $2_{b} \notin f\left(N\left(u_{8}\right)\right)$, then we can recolor $u_{3}$ with $2_{b}$ and $u$ with $2_{a}$ to obtain a coloring satisfying $(a)$ and $(b)$. Thus, we may assume

$$
f\left(N\left(u_{8}\right)\right)=\left\{1_{a}, 2_{a}, 2_{b}\right\} .
$$


By (38), (39), (46), (47), (48), and (49), we have

$$
\left\{1_{a}, 1_{b}, 3_{a}, 3_{b}\right\} \subseteq f\left(N\left(u_{9}\right) \cup N\left(u_{10}\right)-\left\{u_{4}\right\}\right) .
$$

By (50), $1_{b} \notin f\left(N\left(u_{10}\right)-\left\{u_{4}\right\}\right)$, and $2_{b} \notin f\left(B\left(u_{4}, 2\right)-\left\{u_{10}\right\}\right)$ (See Figure 9). Then, we can recolor $u_{10}$ with $1_{b}, u_{4}$ with $2_{b}, u_{1}$ with $1_{b}$, and $u$ with $1_{a}$ to obtain a coloring satisfying $(a)$ and $(b)$.

With Case 2.2.1 handled, from now on by symmetry we may assume

$$
u_{3} u_{5} \notin E(G) \text { and } \quad u_{4} u_{6} \notin E(G) .
$$

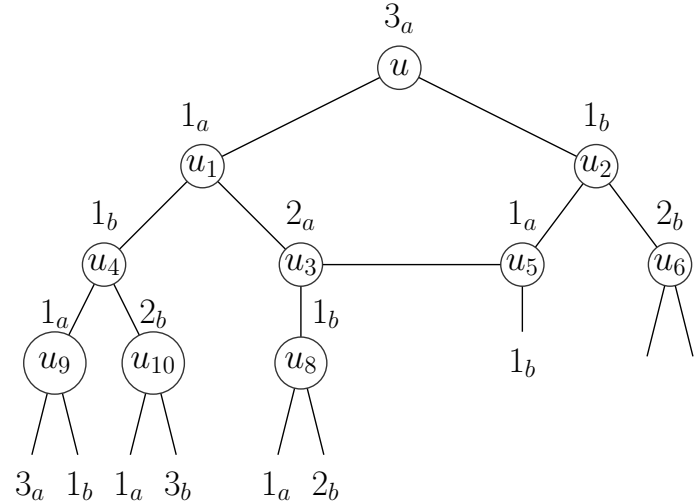

Figure 9: Case 2.2.1.

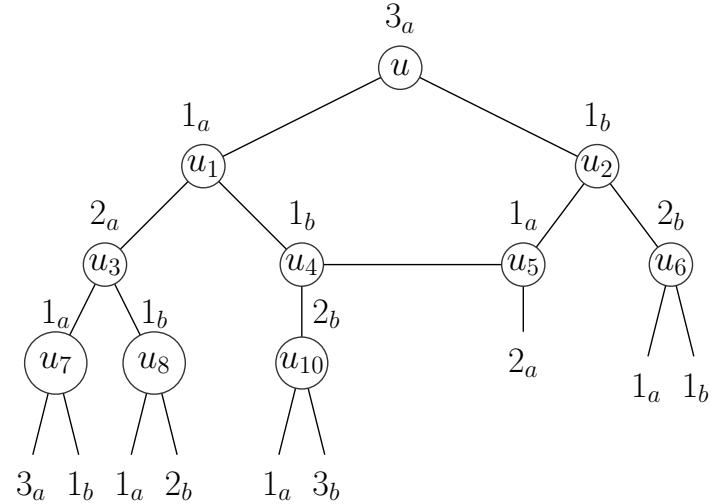

Figure 10: Case 2.2.2.

Case 2.2.2: $\left\{u_{3} u_{5}, u_{4} u_{6}\right\} \cap E(G)=\emptyset$ and $u_{4} u_{5} \in E(G)$, i.e., $u_{9}=u_{5}$. If $2_{a} \notin f\left(N\left(u_{5}\right) \cup N\left(u_{6}\right)\right)$, then we can recolor $u_{2}$ with $2_{a}$ and $u$ with $1_{b}$ to obtain a coloring satisfying $(a)$ and $(b)$. If $1_{b} \notin$ $f\left(N\left(u_{6}\right)-\left\{u_{2}\right\}\right)$ and $2_{b} \notin f\left(N\left(u_{5}\right)-\left\{u_{2}, u_{4}\right\}\right)$, then we can recolor $u_{6}$ with $1_{b}, u_{2}$ with $2_{b}$, and $u$ with $1_{b}$ to obtain a coloring satisfying $(a)$ and $(b)$. With (45), we know

$$
\begin{gathered}
f\left(N\left(u_{5}\right)-\left\{u_{2}, u_{4}\right\}\right)=\left\{2_{a}\right\} \quad \text { and } \quad f\left(N\left(u_{6}\right)-\left\{u_{2}\right\}\right)=\left\{1_{a}, 1_{b}\right\} \\
\text { or } f\left(N\left(u_{5}\right)-\left\{u_{2}, u_{4}\right\}\right)=\left\{2_{b}\right\} \quad \text { and } \quad f\left(N\left(u_{6}\right)-\left\{u_{2}\right\}\right)=\left\{1_{a}, 2_{a}\right\} .
\end{gathered}
$$

If $f\left(N\left(u_{5}\right)-\left\{u_{2}, u_{4}\right\}\right)=\left\{2_{b}\right\}$ and $f\left(N\left(u_{6}\right)-\left\{u_{2}\right\}\right)=\left\{1_{a}, 2_{a}\right\}$, then we recolor $u_{5}$ with $2_{a}, u_{2}$ with $1_{a}$, and $u$ with $1_{b}$ to obtain a coloring satisfying $(a)$ and $(b)$. Thus, we can assume that

$$
f\left(N\left(u_{5}\right)-\left\{u_{2}, u_{4}\right\}\right)=\left\{2_{a}\right\} \quad \text { and } \quad f\left(N\left(u_{6}\right)-\left\{u_{2}\right\}\right)=\left\{1_{a}, 1_{b}\right\} .
$$

If $1_{b} \notin f\left(N\left(u_{7}\right)-\left\{u_{3}\right\}\right)$, then we can recolor $u_{7}$ with $1_{b}$ and it contradicts (37). Thus, we may assume

$$
1_{b} \in f\left(N\left(u_{7}\right)-\left\{u_{3}\right\}\right)
$$

If $1_{a} \notin f\left(N\left(u_{8}\right)-\left\{u_{3}\right\}\right)$, then we can recolor $u_{8}$ with $1_{a}$ and it contradicts (36). If $1_{a} \notin f\left(N\left(u_{10}\right)-\right.$ $\left.\left\{u_{4}\right\}\right)$, then we can recolor $u_{10}$ with $1_{a}$ and it contradicts (37). Therefore, we may assume

$$
1_{a} \in f\left(N\left(u_{10}\right)-\left\{u_{4}\right\}\right) \text { and } 1_{a} \in f\left(N\left(u_{8}\right)-\left\{u_{3}\right\}\right) .
$$

If $2_{b} \notin f\left(N\left(u_{7}\right) \cup N\left(u_{8}\right)-\left\{u_{3}\right\}\right)$, then we can recolor $u_{3}$ with $2_{b}$ and $u$ with $2_{a}$ to obtain a coloring satisfying $(a)$ and $(b)$. Thus, we may assume

$$
2_{b} \in f\left(N\left(u_{7}\right) \cup N\left(u_{8}\right)-\left\{u_{3}\right\}\right) .
$$


By previous arguments, we know that $\left\{3_{a}, 3_{b}\right\} \cap f\left(\left\{u_{2}, u_{3}, u_{4}, u_{5}, u_{6}, u_{7}, u_{8}, u_{10}\right\}\right)=\emptyset$. With (38), (39), and (52), we know that $\left\{3_{a}, 3_{b}\right\} \subseteq f\left(N\left(u_{7}\right) \cup N\left(u_{8}\right) \cup N\left(u_{10}\right)-\left\{u_{3}, u_{4}\right\}\right)$. Moreover, by (53), (54), (55), and symmetry, we may assume that

$$
f\left(N\left(u_{10}\right)-\left\{u_{4}\right\}\right)=\left\{1_{a}, 3_{b}\right\} \text { (See Figure 10). }
$$

But we can recolor $u_{10}$ with $1_{b}, u_{4}$ with $2_{b}, u_{1}$ with $1_{b}$, and $u$ with $1_{a}$ to obtain a coloring satisfying (a) and $(b)$.

Case 2.2.3: $\left\{u_{3} u_{5}, u_{4} u_{6}, u_{4} u_{5}\right\} \cap E(G)=\emptyset$ and $u_{4} u_{7} \in E(G)$, i.e., $u_{7}=u_{9}$. If $1_{a} \notin f\left(N\left(u_{8}\right)-\right.$ $\left.u_{3}\right)$, then we recolor $u_{8}$ with $1_{a}, u_{3}$ with $1_{b}$, and $u$ with $2_{a}$ to obtain a coloring satisfying $(a)$ and $(b)$. Thus, we may assume $1_{a} \in f\left(N\left(u_{8}\right)-u_{3}\right)$. If $1_{a} \notin f\left(N\left(u_{10}\right)-u_{4}\right)$, then we recolor $u_{10}$ with $1_{a}$, $u_{1}$ with $2_{b}$, and $u$ with $1_{a}$ to obtain a coloring satisfying $(a)$ and $(b)$. Thus, we may also assume $1_{a} \in f\left(N\left(u_{10}\right)-u_{4}\right)$. If $2_{b} \notin f\left(N\left(u_{7}\right) \cup N\left(u_{8}\right)-\left\{u_{3}, u_{4}\right\}\right)$, then we recolor $u_{3}$ with $2_{b}$ and $u$ with $2_{a}$ to obtain a coloring satisfying $(a)$ and $(b)$. With (38), (39), and symmetry, we may assume $f\left(N\left(u_{7}\right) \cup N\left(u_{8}\right)-\left\{u_{3}, u_{4}\right\}\right)=\left\{1_{a}, 2_{b}, 3_{a}\right\}$ and $f\left(N\left(u_{10}\right)-u_{4}\right)=\left\{1_{a}, 3_{b}\right\}$ (See Figure 11). We recolor $u_{7}$ with $1_{b}, u_{4}$ with $1_{a}, u_{1}$ with $1_{b}$, and $u$ with $1_{a}$ to obtain a coloring satisfying $(a)$ and $(b)$.

Thus, we may also assume $u_{4} u_{7} \notin E(G)$.

Below we have $\left\{u_{3} u_{5}, u_{4} u_{6}, u_{4} u_{5}, u_{4} u_{7}\right\} \cap E(G)=\emptyset$. Moreover, by the case (Case 2.2),

$$
\left\{u_{3} u_{6}, u_{4} u_{8}, u_{3} u_{9}, u_{3} u_{10}\right\} \cap E(G)=\emptyset .
$$

Therefore, we also have $\left|\left\{u_{i}: i \in[10]\right\}\right|=10$.

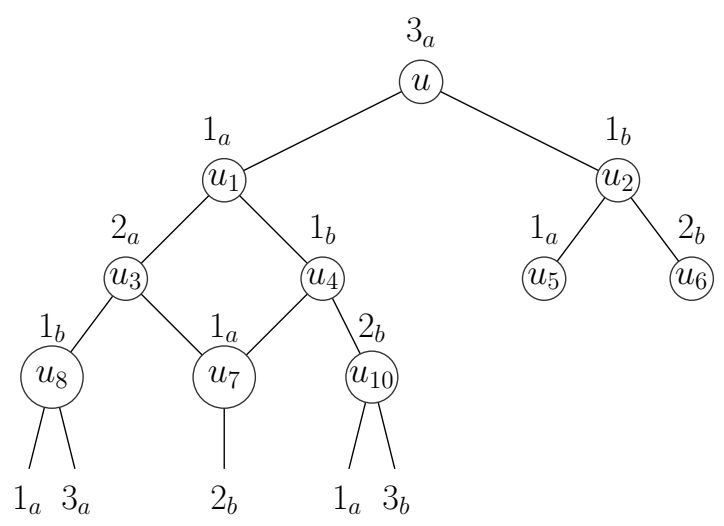

Figure 11: Case 2.2.3.

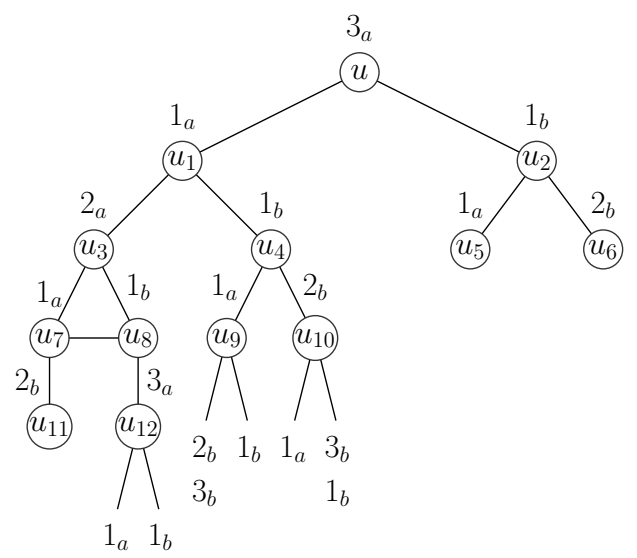

Figure 12: Case 2.2.4.

Case 2.2.4: $u_{7} u_{8} \in E(G)$. By (24) $1_{b} \in f\left(N\left(u_{9}\right)-\left\{u_{4}\right\}\right)$. If $1_{a} \notin f\left(N\left(u_{10}\right)-\left\{u_{4}\right\}\right)$, then we recolor $u_{10}$ with $1_{a}, u_{1}$ with $2_{b}$, and $u$ with $1_{a}$ to obtain a coloring satisfying $(a)$ and $(b)$. Thus, we may assume $1_{a} \notin f\left(N\left(u_{10}\right)-\left\{u_{4}\right\}\right)$. By (38) and (39),$\left\{3_{a}, 3_{b}\right\} \subseteq f\left(N\left(u_{7}\right) \cup N\left(u_{8}\right) \cup N\left(u_{9}\right) \cup N\left(u_{10}\right)\right)$. If $\left\{3_{a}, 3_{b}\right\} \subseteq f\left(N\left(u_{9}\right) \cup N\left(u_{10}\right)\right)$, then $f\left(N\left(u_{9}\right) \cup N\left(u_{10}\right)-\left\{u_{4}\right\}\right)=\left\{1_{a}, 1_{b}, 3_{a}, 3_{b}\right\}, 1_{b} \notin f\left(N\left(u_{10}\right)-\right.$ $\left.\left\{u_{4}\right\}\right)$ and $2_{b} \notin f\left(N\left(u_{9}\right)-\left\{u_{4}\right\}\right)$. Then, we can recolor $u_{10}$ with $1_{b}, u_{4}$ with $2_{b}, u_{1}$ with $1_{b}$, and $u$ with $1_{a}$ to obtain a coloring satisfying $(a)$ and $(b)$. Thus, by symmetry, we can assume

$$
3_{a} \in f\left(N\left(u_{7}\right) \cup N\left(u_{8}\right)-\left\{u_{3}\right\}\right) \quad \text { and } \quad 3_{a} \notin f\left(N\left(u_{9}\right) \cup N\left(u_{10}\right)-u_{4}\right) .
$$


If $2_{b} \notin f\left(N\left(u_{7}\right) \cup N\left(u_{8}\right)-\left\{u_{3}\right\}\right)$, then we recolor $u_{3}$ with $2_{b}$ and $u$ with $2_{a}$ to obtain a coloring satisfying $(a)$ and $(b)$. Thus, we may assume $2_{b} \in f\left(N\left(u_{7}\right) \cup N\left(u_{8}\right)-\left\{u_{3}\right\}\right)$. Let $u_{11} \in N\left(u_{7}\right)-$ $\left\{u_{3}, u_{8}\right\}$ and $u_{12} \in N\left(u_{8}\right)-\left\{u_{3}, u_{7}\right\}$. We may assume

$$
f\left(u_{11}\right)=2_{b} \quad \text { and } \quad f\left(u_{12}\right)=3_{a},
$$

since, by symmetry, the proof for the case $f\left(u_{11}\right)=3_{a}$ and $f\left(u_{12}\right)=2_{b}$ is similar. Note that $3_{a} \notin f\left(B\left(u_{1}, 3\right)-u_{12}\right)$. If $1_{a} \notin f\left(N\left(u_{12}\right)-\left\{u_{8}\right\}\right)$, then we recolor $u_{12}$ with $1_{a}, u_{1}$ with $3_{a}$, and $u$ with $1_{a}$ to obtain a coloring satisfying $(a)$ and $(b)$. If $1_{b} \notin f\left(N\left(u_{12}\right)-\left\{u_{8}\right\}\right)$, then we recolor $u_{12}$ with $1_{b}, u_{8}$ with $1_{a}, u_{7}$ with $1_{b}, u_{1}$ with $3_{a}$, and $u$ with $1_{a}$ to obtain a coloring satisfying $(a)$ and $(b)$. Thus, we may assume

$$
f\left(N\left(u_{12}\right)-\left\{u_{8}\right\}\right)=\left\{1_{a}, 1_{b}\right\} .
$$

If $1_{b} \notin f\left(N\left(u_{11}\right)-\left\{u_{7}\right\}\right)$, then we can recolor $u_{11}$ with $1_{b}, u_{3}$ with $2_{b}$, and $u$ with $2_{a}$ to obtain a coloring satisfying $(a)$ and $(b)$. Thus, we may assume

$$
1_{b} \in f\left(N\left(u_{11}\right)-\left\{u_{7}\right\}\right) \text { (See Figure 12). }
$$

Then, we can recolor $u_{8}$ with $2_{a}, u_{3}$ with $1_{b}$, and $u$ with $2_{a}$ to obtain a coloring satisfying $(a)$ and $(b)$.

Case 2.2.5: $u_{7} u_{8} \notin E(G), u_{8} u_{9} \in E(G)$. Similarly to (48) and (53), we may assume

$$
1_{a} \in f\left(N\left(u_{10}\right)-\left\{u_{4}\right\}\right) \quad \text { and } \quad 1_{b} \in f\left(N\left(u_{7}\right)-\left\{u_{3}\right\}\right) .
$$

If $2_{b} \notin f\left(N\left(u_{7}\right) \cup N\left(u_{8}\right)-\left\{u_{3}\right\}\right)$, then we recolor $u_{3}$ with $2_{b}$ and $u$ with $2_{a}$ to obtain a coloring satisfying $(a)$ and $(b)$. Thus, we may assume $2_{b} \in f\left(N\left(u_{7}\right) \cup N\left(u_{8}\right)-\left\{u_{3}\right\}\right)$. If $1_{b} \notin f\left(N\left(u_{10}\right)-\left\{u_{4}\right\}\right)$ and $2_{b} \notin f\left(N\left(u_{9}\right)-\left\{u_{4}\right\}\right)$, then we can recolor $u_{10}$ with $1_{b}, u_{4}$ with $2_{b}$, $u_{1}$ with $1_{b}$, and $u$ with $1_{a}$ to obtain a coloring satisfying $(a)$ and $(b)$. From (38) and (39), we know that $f\left(N\left(u_{8}\right) \cup N\left(u_{9}\right)-\right.$ $\left.\left\{u_{3}, u_{4}\right\}\right) \subseteq\left\{2_{b}, 3_{a}, 3_{b}\right\}$ (See Figure 13). But it contradicts (24).

Therefore, we may assume $u_{8} u_{9} \notin E(G)$.

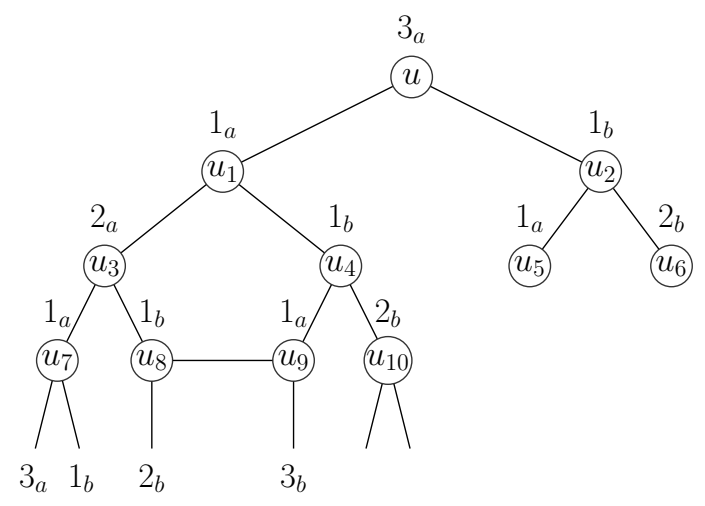

Figure 13: Case 2.2.5.

Case 2.2.6: $u_{7} u_{8} \notin E(G), u_{8} u_{9} \notin E(G)$. If $\left|N\left(u_{7}\right)\right|=\left|N\left(u_{8}\right)\right|=\left|N\left(u_{9}\right)\right|=\left|N\left(u_{10}\right)\right|=3$, then we let

$$
\left\{u_{11}, u_{12}\right\} \subseteq N\left(u_{7}\right)-\left\{u_{3}\right\}, \quad\left\{u_{13}, u_{14}\right\} \subseteq N\left(u_{8}\right)-\left\{u_{3}\right\}, \quad\left\{u_{15}, u_{16}\right\} \subseteq N\left(u_{9}\right)-\left\{u_{4}\right\},
$$


and $\left\{u_{17}, u_{18}\right\} \subseteq N\left(u_{10}\right)-\left\{u_{4}\right\}$.

It is possible that $\left|\left\{u_{i}: i \in[18]-[10]\right\}\right| \neq 8$ or $\left\{u_{5}, u_{6}\right\} \cap\left\{u_{i}: i \in[18]-[10]\right\} \neq \emptyset$, but this will not affect the proof below.

Similarly to (46), (47), (48), (49), we may assume

$$
f\left(u_{12}\right)=f\left(u_{16}\right)=1_{b} \quad \text { and } \quad f\left(u_{13}\right)=f\left(u_{17}\right)=1_{a} .
$$

Similarly to (55) and (56), we may assume

$$
\left\{2_{b}, 3_{a}\right\} \subseteq f\left(N\left(u_{7}\right) \cup N\left(u_{8}\right)-\left\{u_{3}\right\}\right) .
$$

If $1_{b} \notin f\left(N\left(u_{10}\right)-\left\{u_{4}\right\}\right)$ and $2_{b} \notin f\left(N\left(u_{9}\right)-\left\{u_{4}\right\}\right)$, then we can recolor $u_{10}$ with $1_{b}$, $u_{4}$ with $2_{b}$, $u_{1}$ with $1_{b}$, and $u$ with $1_{a}$ to obtain a coloring satisfying $(a)$ and (b). With (39), we may assume

$$
\text { either } f\left(u_{15}\right)=3_{b} \text { and } f\left(u_{18}\right)=1_{b} \text { or } f\left(u_{15}\right)=2_{b} \text { and } f\left(u_{18}\right)=3_{b} \text {. }
$$

If $\left|N\left(u_{11}\right)\right|=\left|N\left(u_{12}\right)\right|=\left|N\left(u_{13}\right)\right|=\left|N\left(u_{14}\right)\right|=3$, then we let $\left\{u_{19}, u_{20}\right\} \subseteq N\left(u_{11}\right),\left\{u_{21}, u_{22}\right\} \subseteq$ $N\left(u_{12}\right),\left\{u_{23}, u_{24}\right\} \subseteq N\left(u_{13}\right),\left\{u_{25}, u_{26}\right\} \subseteq N\left(u_{14}\right)$.

By (62), we have

$$
\text { either } f\left(u_{11}\right)=2_{b} \text { and } f\left(u_{14}\right)=3_{a} \text { or } f\left(u_{11}\right)=3_{a} \text { and } f\left(u_{14}\right)=2_{b} \text {. }
$$

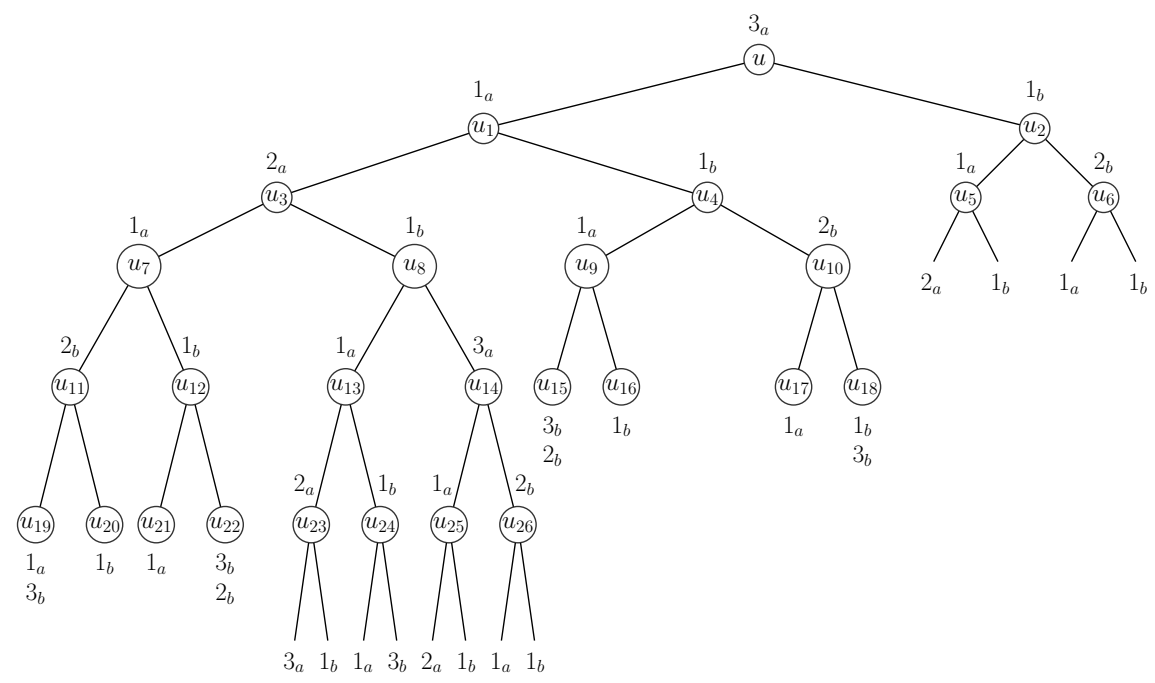

Figure 14: Case 2.2.6.1.

Case 2.2.6.1: $f\left(u_{11}\right)=2_{b}$ and $f\left(u_{14}\right)=3_{a}$. If $1_{b} \notin f\left(N\left(u_{13}\right)-\left\{u_{8}\right\}\right)$, then we can recolor $u_{13}$ with $1_{b}, u_{8}$ with $1_{a}, u_{3}$ with $1_{b}$, and $u$ with $2_{a}$ to obtain a coloring satisfying $(a)$ and $(b)$. If $2_{b} \notin f\left(N\left(u_{13}\right) \cup N\left(u_{14}\right)-\left\{u_{8}\right\}\right)$, then we can recolor $u_{8}$ with $2_{b}, u_{3}$ with $1_{b}$, and $u$ with $2_{a}$ to obtain a coloring satisfying $(a)$ and $(b)$. Thus, we may assume

$$
2_{b} \in f\left(N\left(u_{13}\right) \cup N\left(u_{14}\right)-\left\{u_{8}\right\}\right) .
$$


If $2_{a} \notin f\left(N\left(u_{13}\right) \cup N\left(u_{14}\right)-\left\{u_{8}\right\}\right)$, then we can recolor $u_{8}$ with $2_{a}, u_{3}$ with $1_{b}$, and $u$ with $2_{a}$ to obtain a coloring satisfying $(a)$ and $(b)$. Thus, we may also assume

$$
2_{a} \in f\left(N\left(u_{13}\right) \cup N\left(u_{14}\right)-\left\{u_{8}\right\}\right) .
$$

If $1_{b} \notin f\left(N\left(u_{11}\right)-\left\{u_{7}\right\}\right)$, then we can recolor $u_{11}$ with $1_{b}$ and it contradicts (64). Similarly, $1_{a} \in f\left(N\left(u_{14}\right)-\left\{u_{8}\right\}\right)$. If $1_{a} \notin f\left(N\left(u_{12}\right)-\left\{u_{7}\right\}\right)$, then we can recolor $u_{12}$ with $1_{a}, u_{7}$ with $1_{b}$, and it contradicts (37). Similarly, $1_{b} \in f\left(N\left(u_{13}\right)-\left\{u_{8}\right\}\right)$. Thus, we may assume

$$
\left|N\left(u_{13}\right)\right|=\left|N\left(u_{14}\right)\right|=3, f\left(u_{20}\right)=f\left(u_{24}\right)=1_{b}, \quad \text { and } \quad f\left(u_{21}\right)=f\left(u_{25}\right)=1_{a} .
$$

Furthermore, by (65) and (66), we assume

$$
f\left(u_{23}\right)=2_{a} \text { and } f\left(u_{26}\right)=2_{b},
$$

since the argument for $f\left(u_{23}\right)=2_{b}$ and $f\left(u_{26}\right)=2_{a}$ is similar. If $\left\{1_{a}, 1_{b}\right\} \neq f\left(N\left(u_{26}\right)-\left\{u_{14}\right\}\right)$, then we can recolor $u_{26}$ with a color $x \in f\left(N\left(u_{26}\right)-\left\{u_{14}\right\}\right)-\left\{1_{a}, 1_{b}\right\}$, $u_{8}$ with $2_{b}, u_{3}$ with $1_{b}$, and $u$ with $2_{a}$ to obtain a coloring satisfying $(a)$ and $(b)$. Thus, we may assume

$$
f\left(N\left(u_{26}\right)-\left\{u_{14}\right\}\right)=\left\{1_{a}, 1_{b}\right\} .
$$

If $1_{b} \notin f\left(N\left(u_{25}\right)-\left\{u_{14}\right\}\right)$, then we can recolor $u_{25}$ with $1_{b}, u_{14}$ with $1_{a}$, and it contradicts (64). Thus, we may assume

$$
1_{b} \in f\left(N\left(u_{25}\right)-\left\{u_{14}\right\}\right) .
$$

If $f\left(u_{19}\right) \neq 1_{a}$ and $f\left(u_{22}\right) \neq 2_{b}$, then we can recolor $u_{11}$ with $1_{a}, u_{7}$ with $2_{b}, u_{3}$ with $1_{a}, u_{1}$ with $2_{a}$, and $u$ with $1_{a}$ to obtain a coloring satisfying $(a)$ and $(b)$. If $3_{b} \notin f\left(N\left(u_{11}\right) \cup N\left(u_{12}\right)-\left\{u_{7}\right\}\right)$, then we can recolor $u_{3}$ with $3_{b}$ and $u$ with $2_{a}$ to obtain a coloring satisfying $(a)$ and $(b)$. Thus, we can assume

$$
\text { either } f\left(u_{19}\right)=1_{a} \text { and } f\left(u_{22}\right)=3_{b} \text { or } f\left(u_{19}\right)=3_{b} \text { and } f\left(u_{22}\right)=2_{b} \text {. }
$$

If $2_{a} \notin f\left(N\left(u_{25}\right) \cup N\left(u_{26}\right)-\left\{u_{14}\right\}\right)$, then by (71), we can recolor $u_{14}$ with $2_{a}, u_{3}$ with $3_{a}$, and $u$ with $2_{a}$ to obtain a coloring satisfying $(a)$ and (b). With (69), we may assume

$$
2_{a} \in f\left(N\left(u_{25}\right)-\left\{u_{14}\right\}\right) .
$$

Similarly to (67), we may assume

$$
1_{a} \in f\left(N\left(u_{24}\right)-\left\{u_{13}\right\}\right) \text { and } 1_{b} \in f\left(N\left(u_{23}\right)-\left\{u_{13}\right\}\right) .
$$

If $\left\{3_{a}, 3_{b}\right\} \nsubseteq f\left(N\left(u_{23}\right) \cup N\left(u_{24}\right)\right)$, then we can recolor $u_{8}$ with a color $x \in f\left(N\left(u_{23}\right) \cup N\left(u_{24}\right)\right)-$ $\left\{3_{a}, 3_{b}\right\}, u_{14}$ with $1_{b}, u_{3}$ with $1_{b}$, and $u$ with $2_{a}$ to obtain a coloring satisfying $(a)$ and $(b)$. Therefore,

$$
f\left(N\left(u_{23}\right) \cup N\left(u_{24}\right)-\left\{u_{13}\right\}\right)=\left\{1_{a}, 1_{b}, 3_{a}, 3_{b}\right\} \quad \text { and } \quad 2_{b} \notin f\left(B\left(u_{13}\right)\right) \text { (See Figure 14). }
$$

We recolor $u_{13}$ with $2_{b}, u_{8}$ with $1_{a}, u_{3}$ with $1_{b}$, and $u$ with $2_{a}$ to obtain a coloring satisfying $(a)$ and $(b)$.

Case 2.2.6.2: $f\left(u_{11}\right)=3_{a}$ and $f\left(u_{14}\right)=2_{b}$. Similarly to (67), we may assume

$$
f\left(u_{20}\right)=f\left(u_{24}\right)=1_{b} \quad \text { and } \quad f\left(u_{21}\right)=f\left(u_{25}\right)=1_{a} .
$$




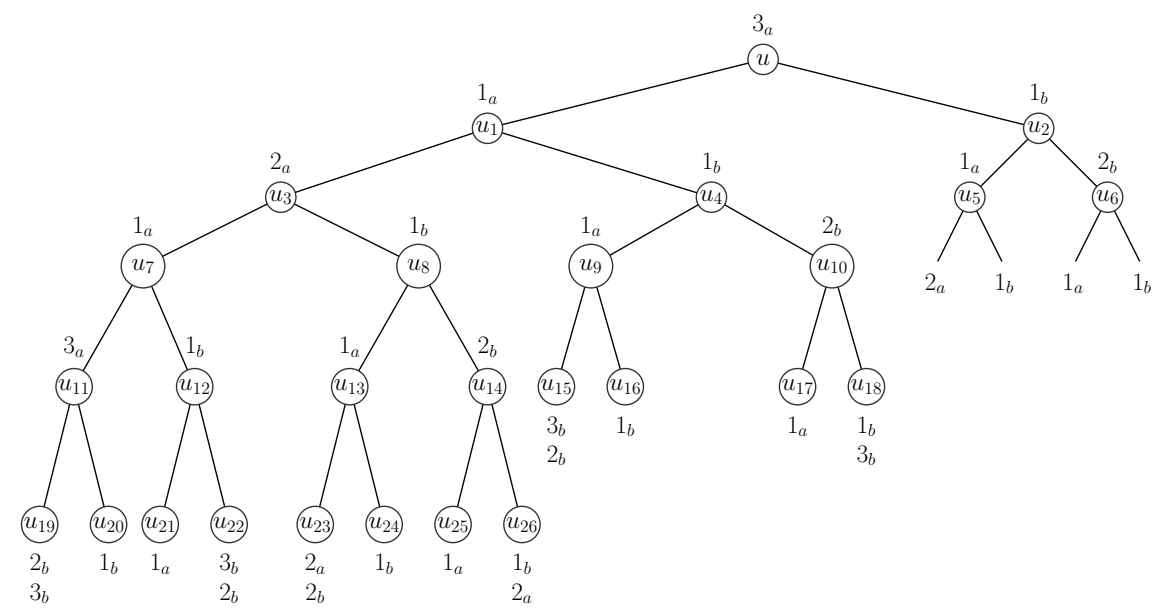

Figure 15: Case 2.2.6.2.

Similarly to (66), we may assume

$$
2_{a} \in f\left(N\left(u_{13}\right) \cup N\left(u_{14}\right)-\left\{u_{8}\right\}\right) .
$$

If $1_{b} \notin f\left(N\left(u_{14}\right)-\left\{u_{8}\right\}\right)$ and $2_{b} \notin f\left(N\left(u_{13}\right)\right)$, then we can recolor $u_{8}$ with $2_{b}, u_{14}$ with $1_{b}, u_{3}$ with $1_{b}$, and $u$ with $2_{a}$ to obtain a coloring satisfying $(a)$ and $(b)$. Thus, we may assume

$$
\begin{gathered}
f\left(N\left(u_{13}\right)-\left\{u_{8}\right\}\right)=\left\{1_{b}, 2_{a}\right\} \quad \text { and } \quad f\left(N\left(u_{14}\right)-\left\{u_{8}\right\}\right)=\left\{1_{a}, 1_{b}\right\} \\
\text { or } \quad f\left(N\left(u_{13}\right)-\left\{u_{8}\right\}\right)=\left\{1_{b}, 2_{b}\right\} \text { and } f\left(N\left(u_{14}\right)-\left\{u_{8}\right\}\right)=\left\{1_{a}, 2_{a}\right\} .
\end{gathered}
$$

If $2_{b} \notin f\left(N\left(u_{11}\right) \cup N\left(u_{12}\right)\right)$, then we can recolor $u_{7}$ with $2_{b}$ and it contradicts (37). If $3_{b} \notin$ $f\left(N\left(u_{11}\right) \cup N\left(u_{12}\right)\right)$, then we can recolor $u_{3}$ with $3_{b}$ and $u$ with $2_{a}$ to obtain a coloring satisfying $(a)$ and $(b)$. Thus, we may assume

$$
f\left(N\left(u_{11}\right) \cup N\left(u_{12}\right)-\left\{u_{7}\right\}\right)=\left\{1_{a}, 1_{b}, 2_{b}, 3_{b}\right\} .
$$

Specifically, we know that $1_{a} \notin f\left(N\left(u_{11}\right)-\left\{u_{7}\right\}\right)$ and $2_{a} \notin f\left(B\left(u_{7}, 2\right)-\left\{u_{3}\right\}\right)$ (See Figure 15). Therefore, we recolor $u_{11}$ with $1_{a}, u_{7}$ with $2_{a}, u_{3}$ with $3_{a}$, and $u$ with $2_{a}$ to obtain a coloring satisfying $(a)$ and $(b)$.

Acknowledgment. We thank Sandi Klavžar, Douglas West, and the referees for their helpful comments.

\section{References}

[1] G. Argiroffo, G. Nasini and P. Torres, The packing coloring problem for lobsters and partner limited graphs, Discrete Appl. Math. 164 (2014), 373-382.

[2] J. Balogh, A. Kostochka and X. Liu, Packing chromatic number of cubic graphs, Discrete Math. 341 (2018), 474-483. 
[3] B. Brešar and J. Ferme, An infinite family of subcubic graphs with unbounded packing chromatic number, Discrete Math. 341 (2018), 2337-2342.

[4] B. Brešar, S. Klavžar and D.F. Rall, On the packing chromatic number of Cartesian products, hexagonal lattice, and trees, Discrete Appl. Math. 155 (2007), 2302-2311.

[5] B. Brešar, S. Klavžar and D.F. Rall, Packing chromatic number of base-3 Sierpiński graphs, Graphs Combin. 32 (2016), 1313-1327.

[6] B. Brešar, S. Klavžar, D.F. Rall and K. Wash, Packing chromatic number under local changes in a graph, Discrete Math. 340 (2017), 1110-1115.

[7] B. Brešar, S. Klavžar, D.F. Rall and K. Wash, Packing chromatic number, (1,1,2,2)-colorings, and characterizing the Petersen graph, Aequationes Math. 91 (2017), 169-184.

[8] B. Brešar, S. Klavžar, D.F. Rall and K. Wash, Packing chromatic number versus chromatic and clique number, Aequationes Math. 92 (2018), 497-513.

[9] J. Czap and S. Jendrol', Facial packing edge-coloring of plane graphs, Discrete Appl. Math. 213 (2016), 71-75.

[10] J. Fiala and P.A. Golovach, Complexity of the packing coloring problem for trees, Discrete Appl. Math. 158 (2010), 771-778.

[11] J. Fiala, S. Klavžar and B. Lidický, The packing chromatic number of infinite product graphs, European J. Combin. 30 (2009), 1101-1113.

[12] N. Gastineau, Dichotomies properties on computational complexity of S-packing coloring problems, Discrete Math. 338 (2015), 1029-1041.

[13] N. Gastineau and O. Togni, S-packing colorings of cubic graphs, Discrete Math. 339 (2016), 2461-2470.

[14] N. Gastineau, P. Holub and O. Togni, On packing chromatic number of subcubic outerplanar graphs, https://arxiv.org/abs/1703.05023.

[15] N. Gastineau, H. Kheddouci and O. Togni, Subdivision into $i$-packing and $S$-packing chromatic number of some lattices, Ars Math. Contemp. 9 (2015), 331-354.

[16] W. Goddard, S.M. Hedetniemi, S.T.Hedetniemi, J.M. Harris and D.F. Rall, Broadcast chromatic numbers of graphs, Ars Combin. 86 (2008), 33-49.

[17] W. Goddard and H. Xu, The S-packing chromatic number of a graph, Discuss. Math. Graph Theory 32 (2012), 795-806.

[18] W. Goddard and H. Xu, A note on S-packing colorings of lattices, Discrete Appl. Math. 166 (2014), 255-262.

[19] D. Korže and A. Vesel, On the packing chromatic number of square and hexagonal lattice, Ars Math. Contemp. 7 (2014), 13-22.

[20] D. Laiche, I. Bouchemakh and É. Sopena, On the packing coloring of undirected and oriented generalized theta graphs, Australas. J. Combin. 66 (2016), 310-329.

[21] D. Laiche, I. Bouchemakh and É. Sopena, Packing coloring of some undirected and oriented coronae graphs, Discuss. Math. Graph Theory 37 (2017), 665-690.

[22] C. Sloper, An eccentric coloring of trees, Austral. J. Combin. 29 (2004), 309-321. 
[23] P. Torres and M. Valencia-Pabon, The packing chromatic number of hypercubes, Discrete Appl. Math. 190-191 (2015), 127-140. 